\title{
Space experimental study on wave modes under instability of thermocapillary convection in liquid bridges on Tiangong-2
}

F

Cite as: Phys. Fluids 32, 034107 (2020); https://doi.org/10.1063/1.5143219

Submitted: 25 December 2019 . Accepted: 25 February 2020 . Published Online: 12 March 2020

Qi Kang (康琦) (D, Di Wu (吴笛) (D, Li Duan (段俐) (D), Jianquan Zhang (张建泉), Bin Zhou (周涁) (D), Jia Wang (王佳) (D), Zhiyi Han (韩志一) (D), Liang Hu (胡良) (D), and Wenrui Hu (胡文瑞)

\section{COLLECTIONS}

F This paper was selected as Featured
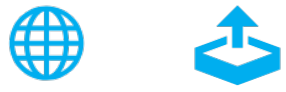

\section{ARTICLES YOU MAY BE INTERESTED IN}

Numerical investigation on formation and motion of bubble or droplet in quiescent flow Physics of Fluids 32, 032106 (2020); https://doi.org/10.1063/1.5143098

Splashing of fuel drops impacting on heated solid surfaces

Physics of Fluids 32, 032104 (2020); https://doi.org/10.1063/1.5139589

Vortex-dynamical implications of nonmonotonic viscous dissipation of off-center droplet bouncing

Physics of Fluids 32, 032004 (2020); https://doi.org/10.1063/5.0003057

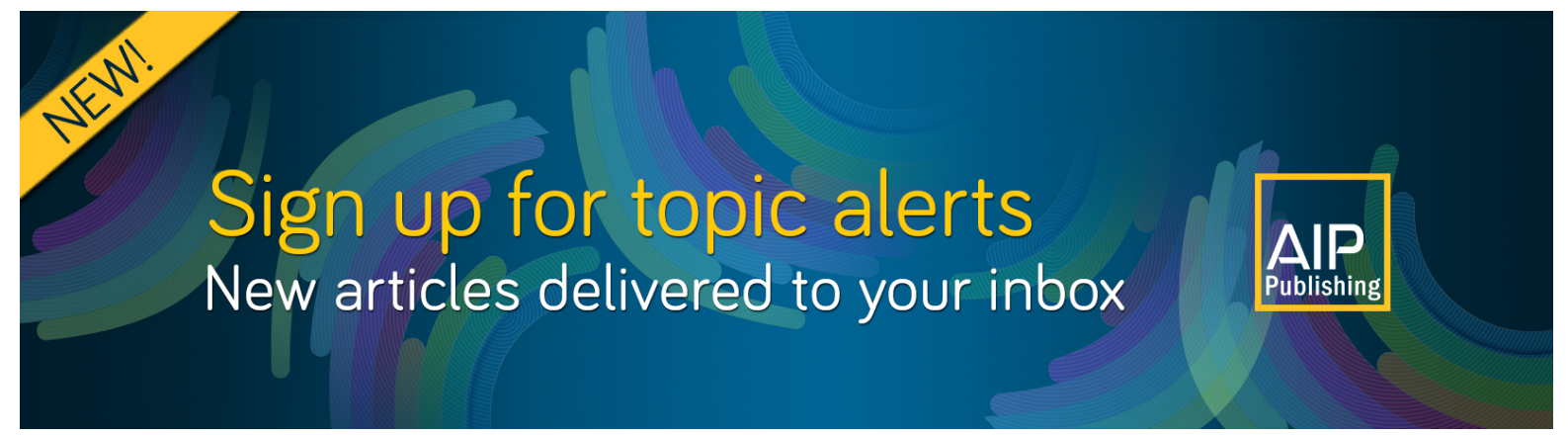




\title{
Space experimental study on wave modes under instability of thermocapillary convection in liquid bridges on Tiangong-2
}

\author{
Cite as: Phys. Fluids 32, 034107 (2020); doi: 10.1063/1.5143219 \\ Submitted: 25 December 2019 - Accepted: 25 February 2020 • \\ Published Online: 12 March 2020
}

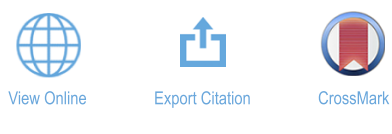

\author{
Qi Kang (康琦), ${ }^{1,2, a)}$ (D) Di Wu (吴笛), (D) Li Duan (段俐), ${ }^{1,2, a)}$ (D) Jianquan Zhang (张建泉), ${ }^{3}$ Bin Zhou (周涁),

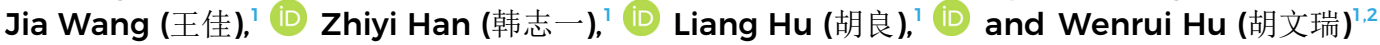

\author{
AFFILIATIONS \\ ${ }^{1}$ Key Laboratory of Microgravity, Institute of Mechanics, Chinese Academy of Sciences, Beijing 100190, \\ People's Republic of China \\ ${ }^{2}$ School of Engineering Sciences, University of Chinese Academy of Sciences, Beijing 100049, People's Republic of China \\ ${ }^{3}$ Technology and Engineering Center for Space Utilization, Chinese Academy of Sciences, Beijing 100094, \\ People's Republic of China
}

a) Authors to whom correspondence should be addressed: kq@imech.ac.cn and duanli@imech.ac.ch

\begin{abstract}
We carried out space experiments on thermocapillary convection in liquid bridges with large Prandtl number in the Tiangong-2 space laboratory, studying the influence of geometrical parameters, including aspect ratio $(A r$, height to diameter) and volume ratio ( $V r)$. It is found that there are two modes of temperature oscillation in thermocapillary convection in liquid bridges: low- and high-frequency oscillation, corresponding to different critical temperature difference and geometrical parameters, respectively. In this paper, the geometrical effect on the wave pattern and its transformation in the oscillating thermocapillary flow is studied in detail. Temperature signals from thermocouples on five measuring spots are analyzed to distinguish different oscillation modes corresponding to characteristics of azimuthal waves, such as wave number, traveling wave, and standing wave. Under a small volume ratio, the low-frequency traveling wave mode transits to the highfrequency standing wave mode; under a large volume ratio, the high-frequency standing wave has abundant mode transformations, such as standing wave $\rightarrow$ traveling wave, standing wave $\rightarrow$ traveling wave $\rightarrow$ standing wave, and so on, and the transformation process of the wave mode is sensitive to the aspect ratio.
\end{abstract}

Published under license by AIP Publishing. https://doi.org/10.1063/1.5143219

\section{INTRODUCTION}

Thermocapillary convection in a liquid bridge is a natural convection driven by the surface tension gradient caused by the temperature difference. With the increase in temperature difference, the transition from steady flow to oscillatory flow occurs at a critical Marangoni number $M a_{\mathrm{c}}{ }^{1,2}$ Oscillatory thermocapillary flow is the main reason for the appearance of striation in the crystal growth, and so studying the flow in a liquid bridge has a significant meaning to improve the quality of the crystal. Scientists have carried out a great deal of research on the critical condition and the mechanism of oscillatory convection. At present, liquid bridges have become an important physics model for the study of thermocapillary instability.
Hydrothermal wave, a widely used explanation for such an oscillatory convection, was first proposed by Smith and Davis ${ }^{3}$ using linear-stability analysis (LSA). The method of LSA was soon used to analyze the thermocapillary instability in liquid bridges. $\mathrm{Xu}$ and Davis $^{4}$ studied an infinite liquid bridge and obtained the hydrothermal waves with azimuthal wave number $m=0$ and $m=1$. The hydrothermal wave of $m=1$ traveled along the direction of surface flows, while the hydrothermal wave of $m=0$ propagated opposite to the direction of surface flows. The existence of hydrothermal waves was validated by the space experiment of Schwabe, ${ }^{5}$ who investigated a long liquid bridge in a sounding rocket and found that the hydrothermal wave of $m=1$ was traveling counter to the flow direction. 
The azimuthal wave number $(m)$ is influenced by the Prandtl number $(P r)$ of the fluid. Xu and Davis ${ }^{4}$ pointed out that, if the heat dissipation on the free surface was neglected, the critical wave number was $m=1$ when $\operatorname{Pr}<62.2$, while the critical wave number changed to $m=0$ when $\operatorname{Pr}>62.2$. Xun et al. ${ }^{6}$ demonstrated that, if the liquid bridge was finite, the critical wave number would transform from $m=2$ to $m=1$ with $\operatorname{Pr}$ being increased to 28. Velten $\mathrm{et} \mathrm{al.}$. validated the existence of $m=0$ wave and plotted a map of transition with the increase in Marangoni number Ma. Frank and Schwabe ${ }^{10}$ observed various oscillatory modes, including the $m=1,2$ rotating wave and pulsating wave. Melnikov et al. ${ }^{8}$ observed the mode of $m=1$ and the mixed mode of $m=1+2$ during the transition to chaos. Mixed mode of $m=0+1$ was discovered by Shevtsova et al. . $^{9}$ However, Frank and Schwabe ${ }^{10}$ claimed that the $m=0$ waves and the obliquely running waves were not observed. Ryzhkov $^{11}$ reckoned that the mode of $m=0$ did not exist, but instead they found a new critical branch with $m=1$. This new branch, which was ignored by $\mathrm{Xu}$ and Davis, ${ }^{4}$ was closer to the result of Schwabe. The azimuthal wave number of hydrothermal waves still has a dispute.

The geometry of a liquid bridge has a significant effect on the stability of thermocapillary convection. Most studies focus on the aspect ratio $(A r)$ effect in a cylindrical liquid bridge (volume ratio $V r=1.00$ ). Preisser ${ }^{12}$ found that the relationship between the wavenumber $\mathrm{m}$ and the aspect ratio $A r$ was $m \times A r=1.1$ through ground experiments. As the aspect ratio decreases, the wavenumber of critical oscillation shows an increasing trend. On the International Space Station (ISS), Japanese scientists conducted space experiments on liquid bridges with different aspect ratios and large Prandtl numbers $\left(\operatorname{Pr}=67\right.$ and 207). ${ }^{13,14}$ They found that the oscillatory flow with $m=1$ was related to a three-dimensional unsteady roll. When 1.00 $\leq A r \leq 1.25$, the roll propagated along the direction of surface flow; when $A r \geq 1.25$, the roll propagated opposite to the direction of surface flow, and the latter was consistent with the hydrothermal waves observed by Schwabe.

$\mathrm{Hu}$ et $a l .{ }^{15}$ first discovered the volume ratio $(V r)$ effect in ground experiments, in which the critical temperature difference is very sensitive to the volume ratio. They pointed out that the critical curve was divided into two branches with a "gap" in the middle, where the flow was steady. Chen and $\mathrm{Hu}^{16}$ carried out linear stability analysis to investigate the volume ratio effect and found that the two-branch theory existed only when the $\operatorname{Pr}$ of the liquid bridge is large. Xun et al. ${ }^{17}$ re-analyzed the stability of the "gap" and suggested that a new oscillation mode with $m=0$ exists in the "gap." Wang et al. ${ }^{18}$ demonstrated that Hu's two-branch theory was available to the liquid bridge with a large diameter through ground experiment. Recently, thermocapillary convection in an annular pool was studied on the SJ-10 satellite by Kang et al. ${ }^{19-21}$ They found that Hu's two-branch theory was also available to the volume ratio effect of an annular pool.

The two branches of the critical curve infer different wave modes. However, which oscillation modes the two branches of critical curve correspond to are still academically controversial. Chen and $\mathrm{Hu}^{16}$ and Emkov ${ }^{22}$ proved by linear-stability analysis that the two branches of the critical curve corresponded to the same azimuthal wave number $m=1(A r=1)$. However, Shevtsova et al. ${ }^{2}$ believed that the two branches corresponded to different azimuthal wave numbers, namely, $m=1$ for a slim liquid bridge and $m=2$ for a fat liquid bridge. Sim and $\mathrm{Zebib}^{24}$ also discovered traveling waves with $m=1$ and $m=2$ through numerical simulation, but they were the opposite to the result of Shevtsova. Masud et al..$^{25}$ put forward that the change of basic flow caused the two branches of the critical curve, and the basic flow in the slim bridge consisted of two pairs of rolls, while the basic flow in the fat bridge consisted of one pair of rolls.

In the work described herein, we have carried out space experiments in China's Tiangong-2 (TG-2) space laboratory to systematically study how oscillatory thermocapillary flow is affected by the geometry of a liquid bridge. ${ }^{26}$ The two-branch theory is expanded from the volume ratio effect to the geometry effect, and two oscillatory zones are discovered in the $V r-A r$ parameter space. The zones correspond to different modes: low-frequency mode and highfrequency mode. The low-frequency mode has a lower critical temperature difference, and it happens in the slender liquid bridge with a small volume ratio or a large aspect ratio; the high-frequency mode has a higher critical temperature difference, and it happens in the dumpy liquid bridge with a large volume ratio or a small aspect ratio. These characteristics are consistent with the two-branch phenomenon discovered by $\mathrm{Hu}$ et al. ${ }^{15}$ The critical condition, the frequency characteristic, and transition of frequency in our space experiment were discussed in Ref. 26. However, the wave number and the spatial characteristics of wave were largely unknown.

In this paper, we studied the wave mode and the wave transformation in the thermocapillary convection of a liquid bridge with a gradual increase in temperature difference. Based on the oscillation measured by thermocouples installed on different spots, the wave characteristics, such as the azimuthal wave number, the traveling wave (TW), and the standing wave (SW), are analyzed depending on the aspect ratio and the volume ratio. This study gives a more clear description of the wave modes, and a map of supercritical transformation is concluded.

\section{SPACE EXPERIMENTS AND DATA ANALYSES}

\section{A. Space experiments}

Space experiments on thermocapillary convection in liquid bridges with large Prandtl number is a project of fluid dynamics experiments carried out in the space laboratory TG-2. The aspect ratio (height to diameter) $\mathrm{Ar}$ and volume ratio $\mathrm{Vr}$ are key parameters in these space experiments. The aspect ratio is defined as $A r=H / D$, where the diameter $D=20 \mathrm{~mm}$ and the height $H=3 \mathrm{~mm}-22 \mathrm{~mm}$, and the aspect ratio is in the range of $0.15-1.10$. The volume ratio is defined as $V r=V / V_{0} . V$ is the volume of liquid, which is calculated by the volume of liquid injection or the $\int \pi r^{2}(z) d z$, where $r(z)$ represents the free surface of a liquid bridge. $V_{0}=\pi D^{2} H / 4$ is the volume of the circular column between the upper and lower bridge columns. The range of volume ratio in our space experiment is $V r=0.45-1.15$. The volume ratio $V r$ can represent the shape of the free surface of the liquid bridge. Studying the joint effect of aspect ratio $A r$ and volume ratio $\mathrm{Vr}$ on the thermocapillary instability is an important feature of our space experiments.

In order to form thermocapillary convection inside the liquid bridge, a temperature gradient in the axial direction is established to generate the surface tension gradient for driving thermocapillary convection. The temperature difference is established by gradually 
heating one end of the liquid bridge and keeping the other end at $1^{\circ} \mathrm{C}$ lower than ambient temperature. The critical Marangoni number $M a_{\mathrm{c}}$ is the critical condition of thermocapillary convection in the liquid bridge, and it is defined as

$$
M a=\frac{\left|\sigma_{T}\right| \Delta T H}{\rho v \kappa},
$$

where $\rho$ is the density, $v$ is the kinematics viscosity, $\sigma_{T}$ is the surface tension temperature coefficient, $\Delta T_{c}$ is the temperature difference, and $\kappa$ is the thermal diffusivity. When the temperature difference exceeds the critical value $\Delta T_{c}$, thermocapillary convection will transit from the steady state [Fig. 1(a)] to the oscillatory state [Fig. 1(b)].

The fluid medium used in the experiments is $5 \mathrm{cS}$ silicone oil, a product of Shin-Etsu, whose Prandtl number is $\operatorname{Pr}=\frac{v}{\kappa}=67$. Since the viscosity of silicone oil decreases with the increase in temperature, the value of viscosity, $\bar{v}$, at the average temperature of a liquid bridge, $T_{m}=T_{0}+\Delta T / 2-1$, is used in the analysis. The fluid viscosity depending on the temperature is provided in the data sheet of Kf-96 silicone oil,

$$
\log _{10} v_{T}=\frac{763.1}{273+T}-2.559+\log _{10} v_{25}
$$

Five thermocouples near the cold plate capture the oscillation signal during the entire process, and both the critical and supercritical transitions can be clearly observed. As shown in Fig. 1(a), the temperature measuring points by thermocouples marked as T1, T2, T3, T4, and T5 are in the plane, which is $2.5 \mathrm{~mm}$ to the cold end, and distributed in azimuth angles of $0^{\circ},-90^{\circ},-180^{\circ},-225^{\circ}$, and $-270^{\circ}$, respectively. The propagation of oscillation generates waves, and the phase of oscillation depends on the measuring spot; therefore, from temperature signals of $T_{1} \sim T_{5}$, the wave modes of flow field can be analyzed.

Wave mode transformation in the liquid bridge occurs as the temperature difference increases. For the liquid bridge at $A r=0.5$ and $V r=0.54$ (experimental results are shown in Fig. 2), the critical temperature difference $\Delta T_{\mathrm{cl}}$ is $14.89^{\circ} \mathrm{C}$. As the temperature difference increases to $\Delta T_{\mathrm{c} 2}$, obvious secondary transition appears. In this paper, spatial wave characteristics of thermocapillary convection are analyzed by phase analysis method together with principal component analysis method, and not only is the transformation mechanism of wave mode that causes the change in oscillation frequency discovered, but also the underlying wave mode transformation at the same frequency is found.

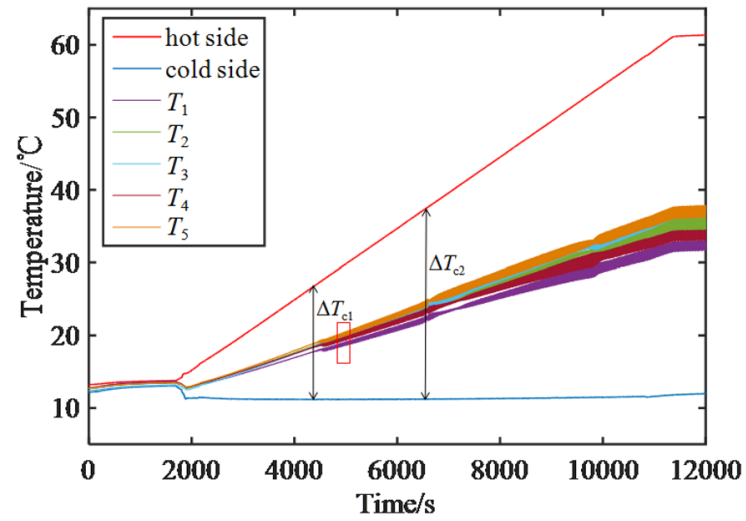

FIG. 2. Thermocouple signals during linear temperature increasing process.

\section{B. Phase analysis of thermocapillary oscillation signals}

There are two probable azimuthal wave modes in oscillatory thermocapillary flow: traveling waves and standing waves. Different wave mode results in the different phase of the oscillations measured by the five thermocouples, and it has different phase characteristics. The oscillation signals in the small window in red color shown in Fig. 2 in the liquid bridge at $A r=0.5$ and $V r=0.54$ are shown in Fig. 3(a), which are obtained by deducting a long-term trend from original temperature signals. Marking the wave trough by dotted lines, we find that the trough passes thermocouples in turn as T5, T4, T3, T2, and T1. Therefore, we judge that this kind of oscillation is a counterclockwise traveling wave. A tiny change in volume ratio may cause the change in critical mode. The oscillation signals of a liquid bridge at $A r=0.5$ and $V r=0.58$ are shown in Fig. 3(b). By drawing lines at time $t_{1}$ and $t_{2}$, we find that T4 and T5 are completely synchronized, and $\mathrm{T} 2$ is opposite to them. We infer that the oscillation is caused by an $m=1$ standing wave where the nodes locate near T1 and $\mathrm{T} 2$ and the amplitudes of $\mathrm{T} 1$ and $\mathrm{T} 2$ are very small. $\mathrm{T} 1$ and $\mathrm{T} 2$ are near the node of standing wave with smaller amplitudes while are double-frequency harmonic oscillations discussed in Sec. III B.

The phase relationship between thermocouple signals is calculated by cross-correlation method as follows:

First, remove the long-term trend in the temperature and normalize the oscillation signals as $\bar{T}_{i}(t)$, select one signal as a reference signal ( $\mathrm{T} 5$ is selected in this paper), and define the phase of reference

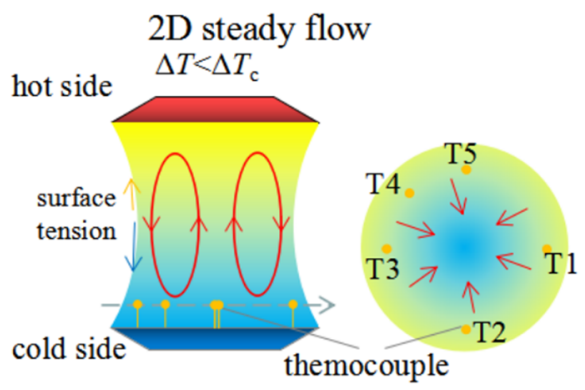

(a)

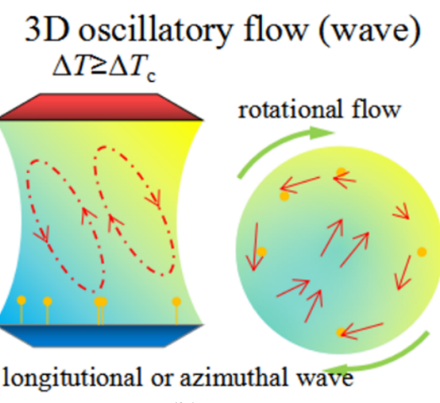

(b)
FIG. 1. Schematic diagram of thermocapillary convection and temperature measuring points in a liquid bridge. (a) 2D steady flow and (b) 3D oscillatory flow (wave). 


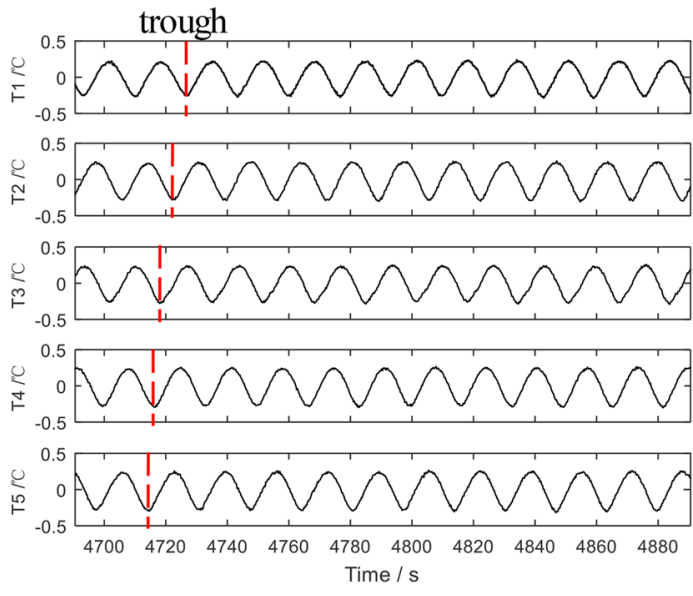

(a)

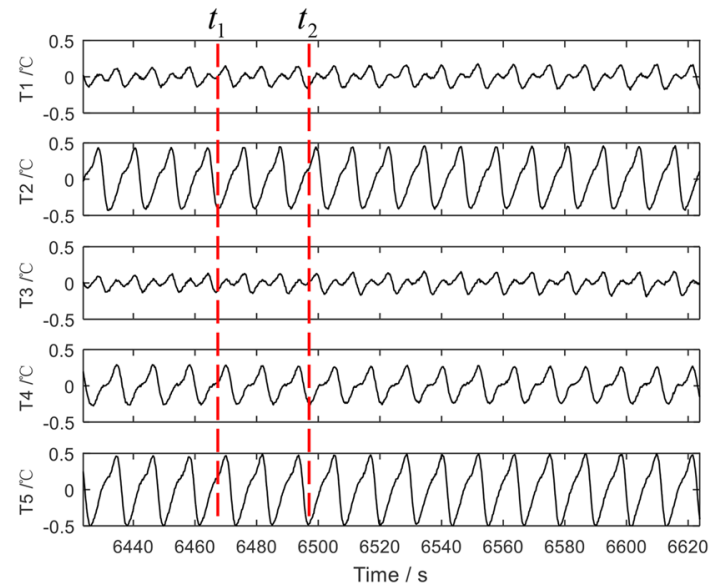

(b)

FIG. 3. Temperature signals from five thermocouples. (a) $A r=0.5, V r=0.54$; (b) $A r=0.5, V r=0.58$.

signal as $0^{\circ}$ or $360^{\circ}$. Then, calculate the cross-correlation function between other signals and the reference signal. At a time $t=t_{0}$, the correlation function between signal $i(i=1,2,3,4,5)$ and signal 5 can be expressed as

$$
R_{i 5}(\tau, t 0)=\int_{t 0-w / 2}^{t 0+w / 2} \bar{T}_{i}(t+\tau) \bar{T}_{5}(t) d t,
$$

where $\mathrm{w}$ is the width of the sampling window, which is set five times larger than the oscillation period. In order to analyze the change of phase with time, we use a moving window to calculate the correlation functions. The time corresponding to the cross-correlation peak value, $\tau_{15}^{\mathrm{p}}$, is the time needed for the oscillation signal propagating from thermocouple 5 to thermocouple $i$. Then, find the main frequency $f_{\mathrm{m}}$ of these signals by Fourier transform. At last, phase differences between signals 1-4 and signal 5 are obtained as

$$
\Delta \varphi_{i 5}=180 \cdot f_{m} \cdot \tau_{i 5}^{p} .
$$

The oscillation of thermocapillary convection can be determined to be a traveling wave or standing wave by phase differences. Table I shows the phase differences of temperature signals shown in Figs. 3(a) and 3(b). In a liquid bridge at $V r=0.54$, the phases of $T_{1}-T_{5}$ are approximately $270^{\circ}, 180^{\circ}, 90^{\circ}, 45^{\circ}$, and $360^{\circ}$, respectively, and it is the phase relationship of a typical counterclockwise traveling wave with $m=1$. In a liquid bridge at $V r=0.58$, the phase differences of the temperature oscillations are approximately $0^{\circ}, 180^{\circ}$, and $360^{\circ}$, and it is a typical standing wave.

TABLE I. Phase difference of thermocouple signals. TW: traveling wave; SW: standing wave.

\begin{tabular}{lccccc}
\hline \hline & $\Delta \varphi_{15}$ & $\Delta \varphi_{25}$ & $\Delta \varphi_{35}$ & $\Delta \varphi_{45}$ & $\Delta \varphi_{55}$ \\
\hline$A r=0.5, V r=0.54$ [Fig. 3(a), TW] & $269^{\circ}$ & $179^{\circ}$ & $96^{\circ}$ & $44^{\circ}$ & $0^{\circ}$ \\
$A r=0.5, V r=0.58$ [Fig. 3(b), SW] & $355^{\circ}$ & $186^{\circ}$ & $172^{\circ}$ & $6^{\circ}$ & $0^{\circ}$ \\
\hline \hline
\end{tabular}

\section{Mode analysis of complex oscillation}

Principal component analysis is a statistical method for dimension reduction. By this dimension reduction technique, independent and unrelated oscillations are extracted from multiple thermocouple signals. In experiments, temperature signals from five thermocouples form a $5 \times n$ signal array, and they are not independent but correlated. For the traveling wave, as an example, the measured signal is related to the position of measuring point $\left(r_{i}, \theta_{i}\right)$ and can be expressed as

$$
\begin{aligned}
X\left(r_{i}, \theta_{i}, 0\right) & =A\left(r_{i}\right) \cos \left[\left(\theta_{i}+\omega t+\psi\right)\right] \\
& =A\left(r_{i}\right)\left[\cos \left(\theta_{i}+\psi\right) \cos \omega t-\sin \left(\theta_{i}+\psi\right) \sin \omega t\right],
\end{aligned}
$$

where $\omega$ and $\psi$ are the angular frequency and the initial phase of the cosine wave.

Any oscillation caused by a sinusoidal traveling wave can be expressed as a linear combination of two orthogonal functions, cos $\omega t$ and $\sin \omega t$. Therefore, suppose there are $\mathrm{m} \times n$ signals, which are time sequences captured $m$ thermocouples, the dimension of signal can be reduced from $\mathrm{m} \times n$ to $2 \times n$ if the wave is a traveling wave.

Similarly, for an ideal standing wave, its signal can always be expressed as

$$
X\left(r_{i}, \theta_{i}, 0\right)=A(r) \cos m \theta_{i} \cos (\omega t+\psi) .
$$

Theoretically, the temperature oscillation of a standing wave only needs one function, $\cos (\omega t+\psi)$, to express; therefore, its dimension can be reduced to a $1 \times \mathrm{n}$ signal array.

By principal component analysis, the signals can be decomposed into five orthogonal components, $\mathrm{w}_{i}(i=1, \ldots, 5)$, and corresponding eigenvectors, $\lambda_{i}(i=1 \ldots 5)$. In physics, $\lambda_{i}$ represents the energy of oscillation component $\mathrm{w}_{\mathrm{i}}$, and it is defined as

$$
\lambda_{i}=\sum_{k=1}^{5}\left(T_{k} w_{i}\right)^{2} \quad(i=1,2, \ldots, 5) .
$$



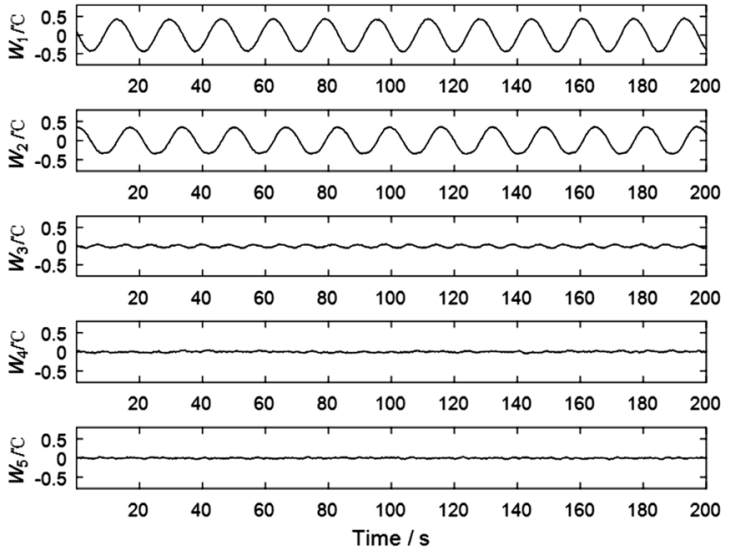

(a)
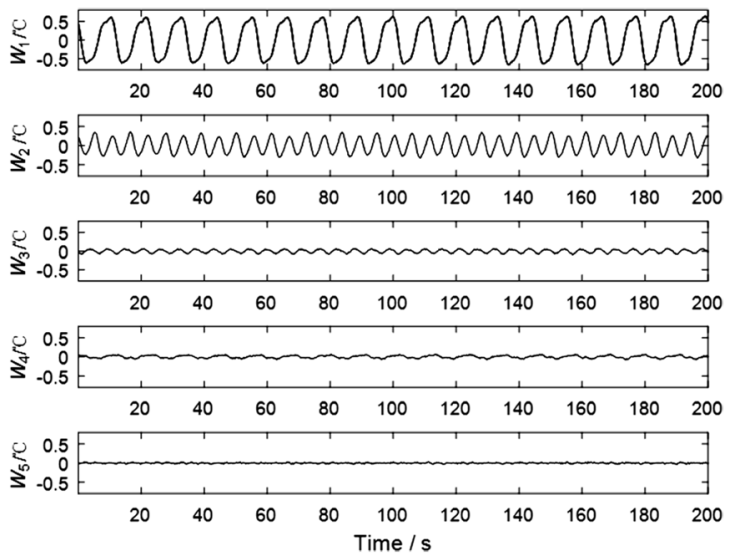

(b)

FIG. 4. Principal component analysis of thermocouple signals. (a) $A r=0.5, V r=0.54$; (b) $A r=0.5, V r=0.58$.

The energy proportion of each principal component, $P_{\mathrm{i}}$, is defined as

$$
P_{i}=\lambda_{i} / \sum_{k=1}^{5} \lambda_{k} \quad(i=1,2, \ldots, 5) .
$$

The modes of traveling wave or standing wave can be judged by energies of principal components. Theoretically, in the standingwave state, the proportion of the first principal component, $P_{1}$, is approximately $100 \%$; in the traveling-wave state, the proportions of the first and second principal components, $P_{1}$ and $P_{2}$, are $50 \%$ each, and with the same frequency and waveform.

Principal component analysis is applied to the critical oscillations in Fig. 3(a) $(A r=0.50$ and $V r=0.54)$ and the oscillations in Fig. 3(b) ( $A r=0.50$ and $V r=0.58)$, respectively. They are decomposed into five orthogonal principal components, $w 1, w 2, \ldots, w 5$, as shown in Figs. 4(a) and 4(b). Table II lists the frequencies, $f_{i}$, eigenvalue, $\lambda_{i}$, and energy proportion, $P_{i}$. When $A r=0.50$ and $V r=0.54$, the first and second principal components have the same frequency, and their energy proportions are $60 \%$ and $39 \%$, respectively. These two components take $99 \%$ of the total energy, and the signals can be dimensionally reduced to a $2 \times \mathrm{n}$ signal array, which conforms to a traveling wave. When $A r=0.50$ and $V r=0.58$, the first principal component constitutes $83.6 \%$, and the flow oscillation appears as a standing wave at this time.
In the $\mathrm{Ar}=0.50$ and $\mathrm{Vr}=0.54$ case, it is found that the second principal component $\mathrm{P} 2$ constitutes $15.1 \%$, which should be 0 in theory. We find that the second principal component is a doublefrequency oscillation of the first principal component. This means that the wave mode is a mixed oscillation of fundamental frequency and double frequency. The advantage of principal component analysis is that the oscillation can be decomposed into different principal components so that the underlying oscillation mode may be discovered. The double frequency oscillation is decomposed from signals by principal component analysis, and it is found to be a new wave mode with $\mathrm{m}=0$ (see Sec. III B).

\section{OSCILLATION MODES}

\section{A. Traveling wave and standing wave with $\mathrm{m}=1$}

The azimuthal wave number, $m$, is a key parameter in representing oscillatory thermocapillary flow, and it is influenced by parameters such as the aspect ratio Ar, Prandtl number, and Biot number. The fluid medium in our space experiments is $5 \mathrm{cSt}$ silicone oil with $\operatorname{Pr}=69$, and the wave number given by linear stability analysis is $m=1$. Wang et al. ${ }^{18}$ found through ground experiments that the wave number $m$ in a short bridge is affected by the volume ratio. Shevtsova et al. ${ }^{23}$ considered that the oscillation in a liquid bridge at

TABLE II. Principal component analysis of thermocouple signals. TW: traveling wave; SW: standing wave.

\begin{tabular}{lcccccc}
\hline \hline & & w1 & w2 & w3 & w4 & w5 \\
\hline$A r=0.5$ & Frequency $f_{i}(\mathrm{~Hz})$ & 0.0585 & 0.0585 & 0.1220 & 0.0146 & 0.1220 \\
$V r=0.54$ & $\lambda_{i}$ & 0.0930 & 0.0613 & 0.0009 & 0.0003 & 0.0002 \\
$(\mathrm{TW})$ & $P_{i}(\%)$ & 59.73 & 39.37 & 0.58 & 0.19 & 0.13 \\
\hline$A r=0.5$ & Frequency $f_{i}(\mathrm{~Hz})$ & 0.0878 & 0.1707 & 0.1707 & 0.0829 & 0.2537 \\
$V r=0.58$ & $\lambda_{i}$ & 0.1721 & 0.0311 & 0.0014 & 0.0010 & 0.0002 \\
$(\mathrm{SW})$ & $P_{i}(\%)$ & 83.62 & 15.11 & 0.68 & 0.49 & 0.10 \\
\hline \hline
\end{tabular}


a small volume ratio is the mode of $m=1$, and the oscillation in a liquid bridge at a large volume ratio is the mode of $m=2$; therefore, the difference in azimuthal wave number $m$ leads to the two branches of critical curve. However, linear stability analyses of Xun et al. ${ }^{6}$ and Ermakov ${ }^{22}$ indicated that both branches correspond to the mode of $m=1$. Therefore, it is necessary to study the azimuthal wave number under different aspect ratios and volume ratios.

In polar coordinates, the azimuthal traveling wave is approximately expressed in terms of trigonometric functions as

$$
X(r, \theta, z)=A(r) \cos [(m \theta+k z+\omega t+\psi)] .
$$

According to stability theory and the space experiment by Schwabe, ${ }^{5}$ the propagation direction of hydrothermal wave is along a tilt direction, that is, $m \neq 0$ and $k \neq 0$. However, the azimuthal wave mode has been discovered in the ground experiments on short liquid bridges, that is, $m \neq 0$ and $k=0$. Although we cannot decide the axial wave number, $k$, we can decide the azimuthal wave number, $m$, from phase differences of signals. As shown in Fig. 3(a), in the liquid bridge at $A r=0.5$ and $V r=0.54$, the phases of signals $T_{1} \sim T_{5}$ obtained through correlation are $269^{\circ}, 179^{\circ}, 96^{\circ}, 44^{\circ}$, and $0^{\circ}$ in turn. The azimuthal angle difference between thermocouple measuring points $1,2,3$, and 5 is $\Delta \theta=90^{\circ}$, and the adjacent phase difference, $\Delta \phi$, is approximately $-90^{\circ}$, with phase differences between measuring points $1-2,2-3,3-5$, and $5-1$ being $\Delta \phi_{12}=-90^{\circ}, \Delta \phi_{23}=-83^{\circ}$, $\Delta \phi_{35}=-96^{\circ}$, and $\Delta \phi_{51}=-91^{\circ}$, respectively. The azimuthal wave number is equal to the ratio of phase difference to azimuthal angle of signals, so $m=|\Delta \phi / \Delta \theta|=1$. As a result, when $A r=0.5$ and $V r=0.54$, the oscillatory thermocapillary flow is a traveling wave mode with $m=1$ [Fig. 5(a)].

A standing wave can be interpreted as the superimposition of a clockwise wave and a counter-clockwise wave,

$$
\begin{aligned}
X(r, \theta, z)= & A(r)\{\cos [(m \theta+k z+\omega t+\psi)] \\
& +\cos [(-m \theta+k z+\omega t+\psi)]\} .
\end{aligned}
$$

By sum-to-product formulas of trigonometric function, we get

$$
X(r, \theta, z)=A(r) \cos m \theta \cos (k z+\omega t+\psi) .
$$

As can be seen in Eq. (5), on the standing wave, the phase difference between any two signal points on the same horizontal section is $0^{\circ}$ or $180^{\circ}$, and the amplitude of signal, $A(r) \cos m \theta$, varies with the change of azimuthal angle, $\theta$. For the oscillatory thermocapillary flow in the liquid bridge at $A r=0.5$ and $V r=0.58$, the phases of $T_{1} \sim T_{5}$ are $355^{\circ}, 186^{\circ}, 172^{\circ}, 6^{\circ}$, and $0^{\circ}$, and the positions of wave nodes are judged to be in between T1 and T2 as well as T3 and T4. For the standing wave with $m=1$, there are two wave nodes with minimum amplitude and two wave loops with maximum amplitude. By signal amplitudes in Fig. 3(b), it is judged that the positions of wave nodes are approximately at T1 and T3; the positions of wave loops are approximately at T2 and T5. Therefore, this oscillatory thermocapillary flow is a standing wave mode with $m=1$ [Fig. 5(b)].

\section{B. Harmonic mode with $\boldsymbol{m}=0$}

On an ideal standing wave with $m=1$, there are two wave loops and two wave nodes, and the amplitudes of wave nodes should be zero. However, the oscillation amplitudes at the nodes [such as $T_{1}$ and $T_{3}$ in Fig. 3(b)] are not zero, and a double-frequency oscillation appears there. From principal component analysis, as shown in Fig. 4(b), the component $w_{1}$ is the oscillation with the $m=1$ standing wave and the frequency is $0.0878 \mathrm{~Hz}$. The components $w_{2}$ and $w_{3}$ are double-frequency oscillation modes whose frequencies are $0.1707 \mathrm{~Hz}$. These two kinds of principal component are transferred into two different physical oscillations, as shown in Fig. 6.

Figure 6(a) shows the physical oscillations of component $w_{1}$. It is an ideal oscillation signals of standing wave with $m=1$, and their phases are $0^{\circ}, 186^{\circ}, 186^{\circ}, 0^{\circ}$, and $360^{\circ}$. Figure $6(\mathrm{~b})$ shows the physical oscillations of components $w_{2}$ and $w_{3}$. The amplitudes of this double-frequency oscillation are $0.17^{\circ} \mathrm{C}, 0.46^{\circ} \mathrm{C}, 0.21^{\circ} \mathrm{C}, 0.18^{\circ} \mathrm{C}$, and $0.26^{\circ} \mathrm{C}$ in the five thermocouple signals. Double-frequency oscillations exist not only at wave nodes but in the entire flow field. By phase analysis on double-frequency signals, it is found that the phase differences of five thermocouple signals are $0^{\circ}$. Therefore, the double-frequency oscillation is an oscillation mode with $m=0$, and temperature oscillations on a circumference reach their maximum value or minimum value in synchronization.

The physical meaning of the $m=0$ wave is a $2 \mathrm{D}$ axisymmetric wave that the oscillations at different azimuth positions are synchronized. There are two possible wave forms, which are radial wave and axial wave. According to the present arrangement of thermocouples, it is difficult to demonstrate which wave form it is. The wave form can be verified by numerical simulation or linear stability analysis. The linear stability analysis by Smith and Davis ${ }^{3}$ shows that the $m=0$ wave is traveling wave from the cold end to the hot end, which may be available to this $m=0$ mode. The $m=0$ mode only occurs in the liquid bridge with small volume ratio, while it cannot be observed at

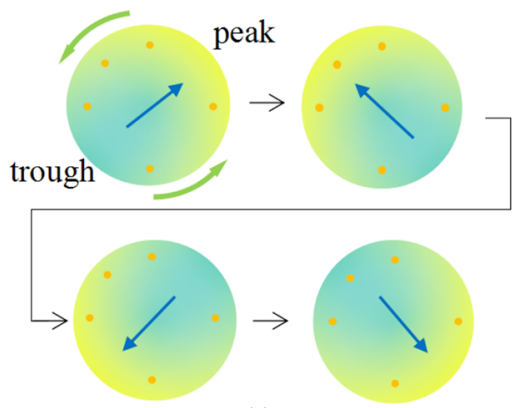

(a)

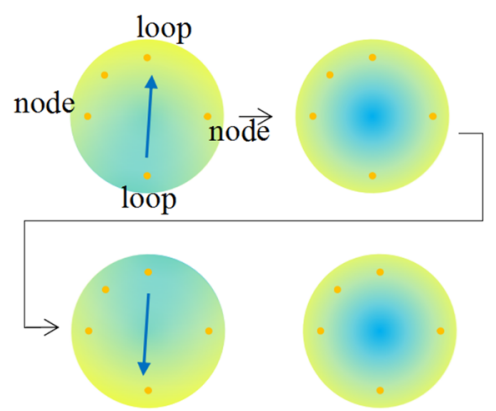

(b)
FIG. 5. Schematic diagrams of traveling wave and standing wave with $m=1$. (a) Traveling wave $(A r=0.5, V r=0.54)$. (b) Standing wave $(A r=0.5, V r=0.58)$. 


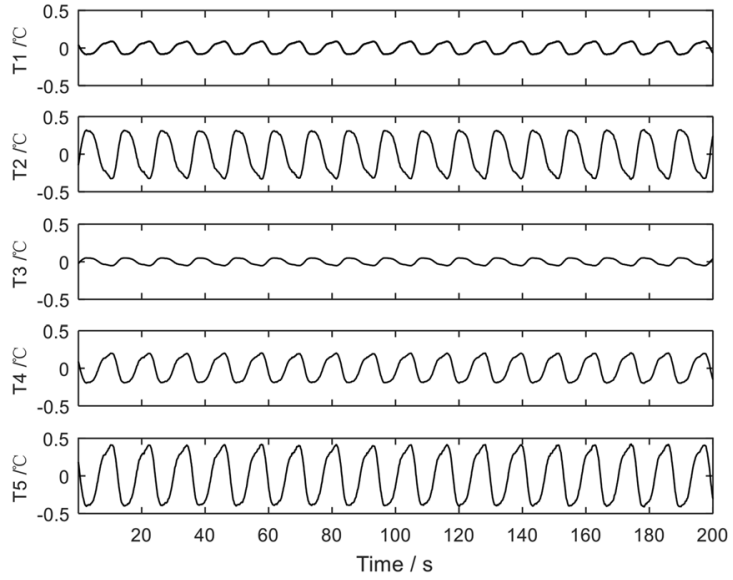

(a)

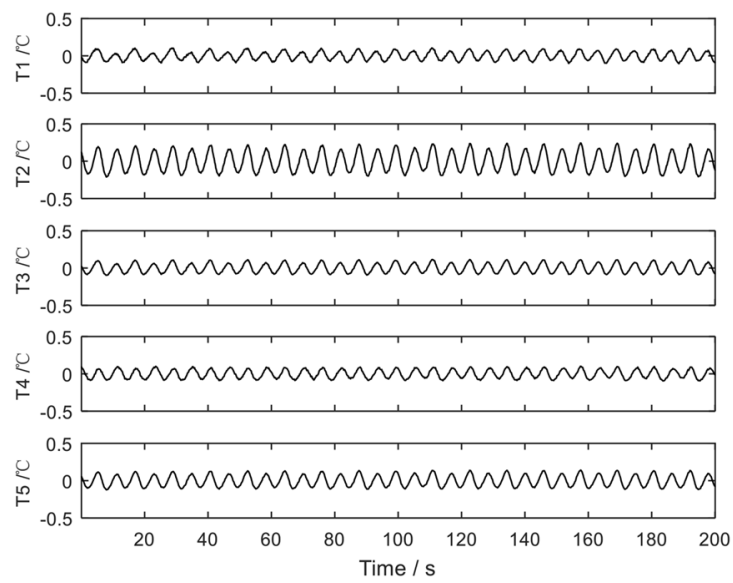

(b)

FIG. 6. Decomposition of principal components of standing wave signals in Fig. 3(b). (a)

(a) Oscillation signals of component $w_{1}$. (b) Oscillation signals of components $w_{2}$ and $w_{3}$

a large volume ratio in the liquid bridge with $A r=0.5,0.6,0.7,0.9$, and 1.0. This supports Ryzhkov's theory ${ }^{11}$ that the $m=0$ wave does not happen in the critical instability of a cylindrical liquid bridge $(V r=1)$. The mixed mode of $m=0+1$ wave found by Shevtsova et al. ${ }^{9}$ may be different from the present work, because they found that the $m=0$ is the wavenumber of the critical instability. Therefore, the harmonic mode of the $m=1$ standing wave is a double-frequency $m=0$ wave in the liquid bridges with small volume ratio.

\section{Harmonic mode with $\boldsymbol{m}=\mathbf{2}$}

In a liquid bridge at a small volume ratio, after a standing wave with $m=1$ has transformed into a traveling wave with $m=1$, the double-frequency harmonic mode will appear in the traveling wave. For example, in the liquid bridge at $A r=0.5$ and $V r=0.7$, when the critical temperature difference of $16.6^{\circ} \mathrm{C}$ is reached, the critical oscillation is the high-frequency standing wave mode with $m$
$=1$. When $\Delta T \geq 25.9^{\circ} \mathrm{C}$, the high-frequency mode transforms from the standing wave to the traveling wave. Figure 7 (a) shows oscillation signals after the standing wave has transformed into the traveling wave (temperature difference $\Delta T=30.8^{\circ} \mathrm{C}$ ). The amplitudes and waveforms of all thermocouple signals are almost exactly the same, and this is a typical traveling wave signal. The fundamental frequency is $0.0781 \mathrm{~Hz}$; peak-to-peak values are $1.45^{\circ} \mathrm{C}, 1.74^{\circ} \mathrm{C}$, $2.02^{\circ} \mathrm{C}, 2.38^{\circ} \mathrm{C}$, and $1.87^{\circ} \mathrm{C}$; and phase relations are $275^{\circ}, 182^{\circ}, 92^{\circ}$, $38^{\circ}$, and $0^{\circ}$.

From Fig. 7(a), we can see that one main peak can be subdivided into two sub-peaks with obvious characteristics of double-frequency oscillation. The harmonic mode in the traveling wave with $m=1$ has characteristics of traveling wave. It is found in experiments that the signals measured by thermocouples at different positions have the same waveform, which means that the azimuthal traveling wave retains its waveform unchanged in azimuthal propagation. Therefore, the azimuthal mode can be reflected by the waveform in a

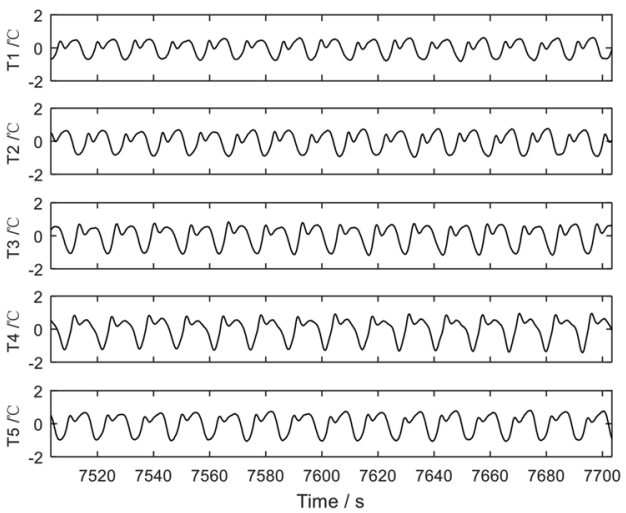

(a)

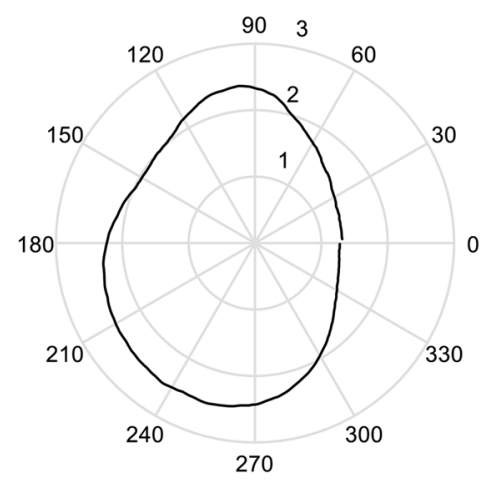

(b)
FIG. 7. Signals of traveling wave and its azimuthal waveform. (a) Traveling wave with $m=1$ superimposed with the harmonic mode with $m=2$. (b) Azimuthal waveform. 
period, as shown in Fig. 7(b). It is clearly observed that this azimuthal wave mode with $m=1$ has characteristics of an azimuthal wave mode with $m=2$.

In fact, any traveling wave is not a sine or cosine mode with a unique wavenumber in the azimuthal direction, but can be considered as a superimposition of sine or cosine modes with $m=1,2,3$, ... If the energy of a harmonic wave is not high enough, there is only one peak and one trough in a period, and this oscillation is shown in Fig. 3(a), in which the wave mode is a traveling wave with $m=1$. If the phenomenon of two sub-peaks appears, as shown in Fig. 7, we believe that the traveling wave at this time is predominated by the $m=1$ traveling wave and mixed with the double-frequency mode with $m=2$. This double-frequency traveling wave with $m=2$ occurs at the transition from the standing wave $(\mathrm{m}=1$, low-frequency mode) to the traveling wave ( $\mathrm{m}=1$, high-frequency mode).

\section{TRANSFORMATION PROCESSES OF WAVE MODES}

\section{A. Oscillation characteristics and transformations of low-frequency mode and high-frequency mode}

Hu et al. ${ }^{15,16}$ put forward the theory of volume ratio effect first as shown in Fig. 8(a), where the marginal curves were separated into two branches with a stable gap. This conclusion was supported by previous studies by $\mathrm{Hu}$ et al., ${ }^{15}$ Wang et al., ${ }^{18}$ and Shevtsova et al. ${ }^{27}$ However, Sumner ${ }^{28}$ and Masud et al. ${ }^{25}$ opposed the existence of "gap," and they ascribed the "gap" phenomenon to the experiment limitations. Sakurai et al. ${ }^{29}$ discovered that the gap was formed in $1 \mathrm{~g}$ condition but absent in $0 \mathrm{~g}$ condition (in drop tower). In our space experiment, the marginal curve exhibits novel configuration, the two branches are intersectant, the left branch is a "J" type, and the right branch extends into the left branch. The intersection of the two branches increases with the aspect ratio. Through the careful analysis, we believe that this result is the expansion of the volume ratio effect proposed by $\mathrm{Hu}$. It provides important issues for the future study of thermocapillary instability in a liquid bridge.

The two-branch phenomenon has been found in our space experiments when the aspect ratio $A r \geq 0.5$. The two branches of critical curve correspond to a low-frequency oscillation mode and a high-frequency oscillation mode, respectively. The low-frequency oscillation is the mode of the left-branch whose critical value is small, while the high-frequency oscillation is the mode of the right branch whose critical value is large and decreases with the increase in volume ratio. With an increase in temperature, the curves of critical condition for the transition of thermocapillary convection in the liquid bridge from steady flow to oscillatory flow, shown as pink dotted lines in Fig. 8(b), are marked as the first critical temperature difference. There should be a volume ratio $V r_{\mathrm{c} 1}$, which is exactly at the demarcation point between the low-frequency mode and the highfrequency mode, where the critical temperature difference and oscillation frequency have jump phenomena with the change in volume ratio.

Taking the liquid bridge at the aspect ratio $A r=0.5$ as an example, Fig. 9 shows the critical temperature difference with the change

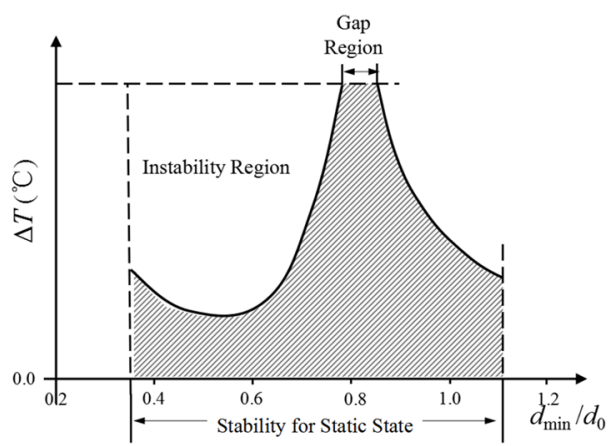

(a)

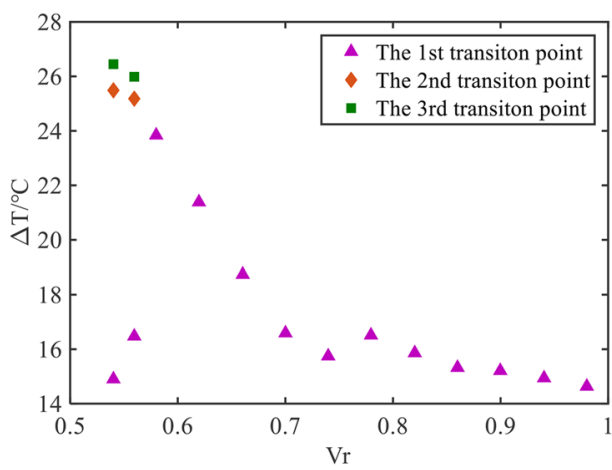

(a)

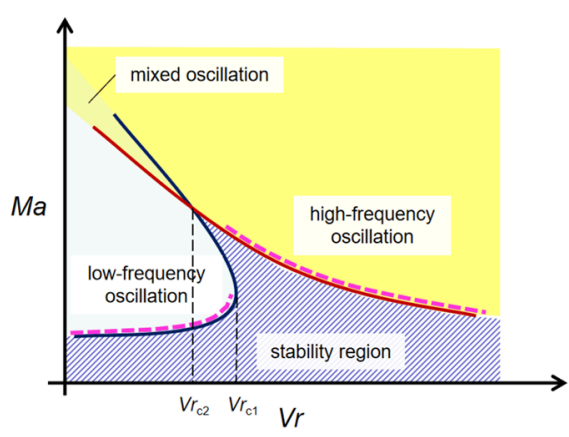

(b)

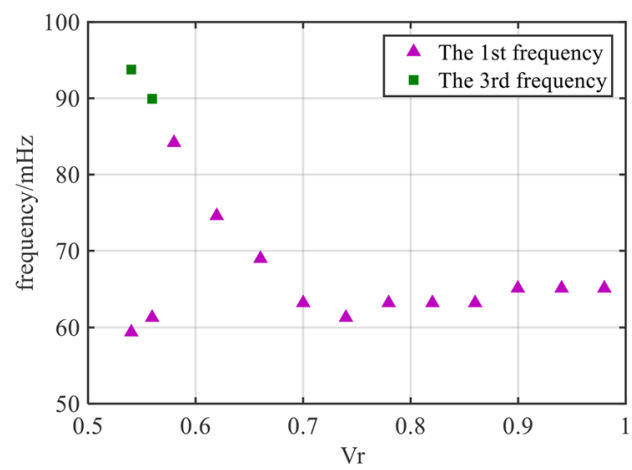

(b)
FIG. 8. Curves of volume ratio effect. (a) Volume ratio effect by $\mathrm{Hu}$ et al. ${ }^{1}$ (b) Volume ratio effect by TG-2 space experiments.
FIG. 9. Critical temperature difference and oscillation frequency at $A r=0.5$. (a) Critical temperature difference. (b) Oscillation frequency. 
in volume ratio. The volume ratio at the demarcation point between the low-frequency mode and the high-frequency mode is $V r_{\mathrm{c} 1}$ $=0.56-0.58$. Near this volume ratio, both the critical temperature difference and oscillation frequency have jump phenomena. When the volume ratio is $V r=0.54$, the critical temperature difference is $14.89^{\circ} \mathrm{C}$, and the oscillation frequency is $59.3 \mathrm{mHz}$. Besides significant differences in the critical temperature difference and oscillation frequency, the wave characteristics are distinct. In the liquid bridge at $A r=0.5$ and $V r=0.54$, as shown in Fig. 3(a), the low-frequency mode is an azimuthal traveling wave with $m=1$; in the liquid bridge at $A r=0.5$ and $V r=0.58$, as shown in Fig. 3(b), the high-frequency mode is an azimuthal standing wave with $m=1$. Therefore, the critical oscillations of the low-frequency mode and the high-frequency mode correspond to the traveling wave $(m=1)$ and the standing wave $(m=1)$, respectively.

Figure 10(a) shows the phase relationship between the thermocouple signals at the onset of oscillation. In liquid bridges at $V r=0.54$ and 0.58 , the phases of $T_{1}-T_{5}$ are about $270^{\circ}, 180^{\circ}, 90^{\circ}$, $45^{\circ}$, and $360^{\circ}$. This is the phase relationship of a typical counterclockwise traveling wave with $m=1$. When $V r \geq 0.62$, the phase differences of temperature oscillation are about $0^{\circ}, 180^{\circ}$, and $360^{\circ}$, and this is a typical standing wave oscillation. Figure 10 (b) shows proportions of principal components of critical oscillation at different volume ratios. When $V r \leq 0.56$, the first principal component takes about $60 \%$, and the flow oscillation appears as a traveling wave. When $V r \geq 0.62$, the first principal component takes about $90 \%$, and the flow oscillation appears as a standing wave. Therefore, the wave number of the liquid bridge at $A r=0.5$ is $m=1$, and the critical wave is the traveling wave when $V r<V r_{\mathrm{c} 1}$, while it is the standing wave when $V r>V r_{\mathrm{c} 1}$.

Critical conditions and critical oscillation modes are mainly affected by geometric parameters such as the aspect ratio and the volume ratio of a liquid bridge. Figure 11(a) shows the distribution map of critical oscillation period in $V r-A r$ space, where the lowfrequency mode and the high-frequency mode correspond to Zone 1 and Zone 2, respectively. Figure 11(b) shows the distribution map of critical oscillation mode, where Zone 1 corresponds to the traveling wave with $m=1$, and Zone 2 corresponds to the standing wave with $m=1$. The demarcation line of the two zones in $V r-A r$ space is approximately a straight line: $\mathrm{Ar}-3.2 \mathrm{Vr}+1.4=0$. Therefore, the critical wave mode can be judged from the geometric parameters in experiments.

With the increase in temperature difference, the low-frequency oscillation mode (in Zone1) is unstable and will transit to the high-frequency oscillation mode. This transition of frequency can be explained by the competition of the two branches, as shown in Fig. 8(b), when $V r<V r_{\mathrm{cl}}$. From the view of azimuthal wave mode, the transition of frequency leads to a transformation from the

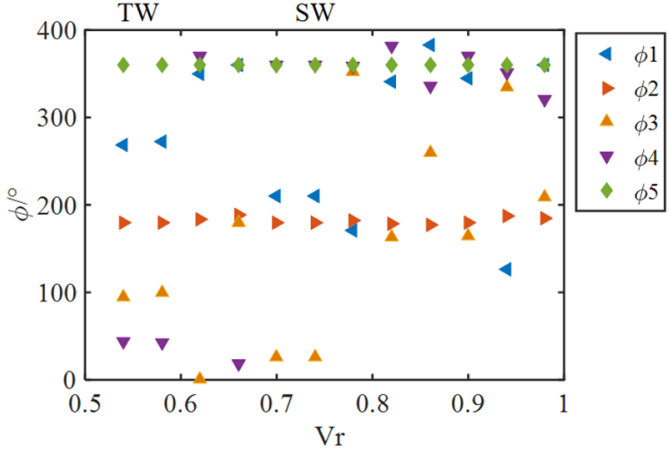

(a)

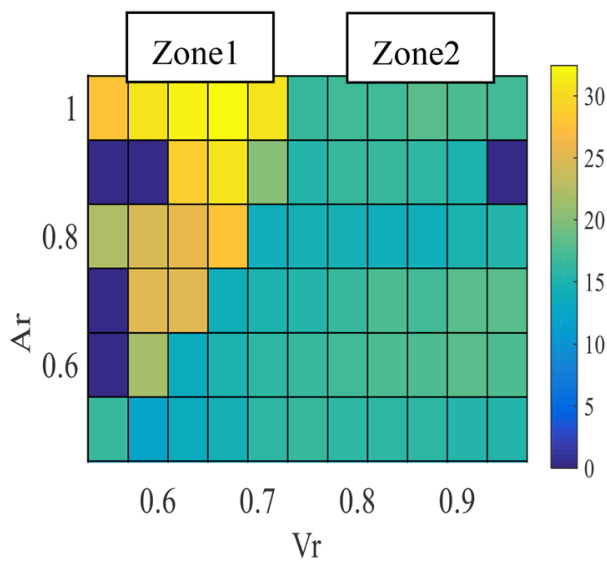

(a)

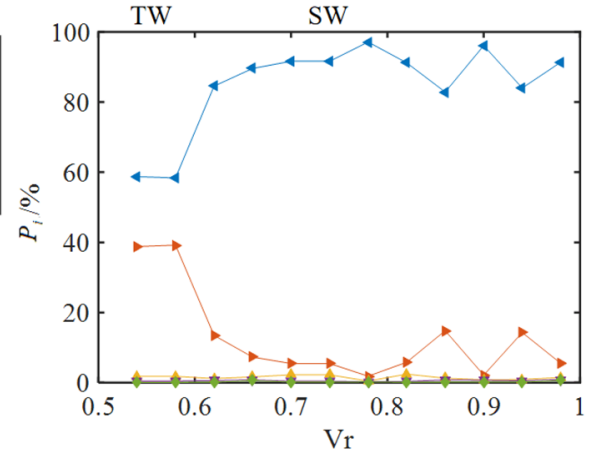

(b)

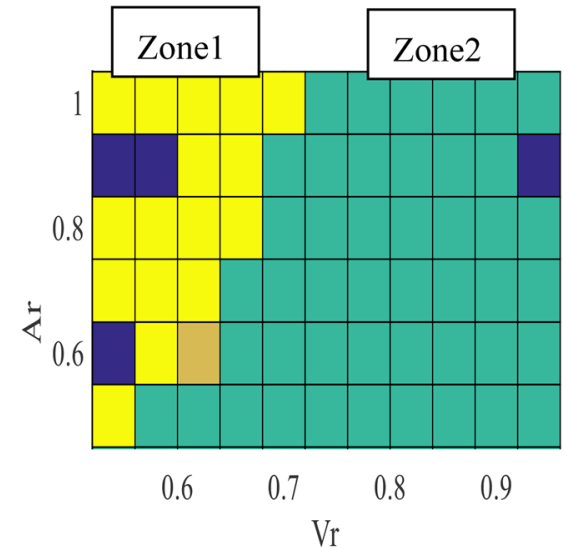

(b)
FIG. 10. Phase of critical oscillation and proportion of principal component vs $\mathrm{Vr}$ at $A r=0.5$. (a) Phases of five thermocouple signals vs volume ratio Vr. (b) Proportions of principal components $P_{i}$ vs volume ratio $\mathrm{Vr}$. 

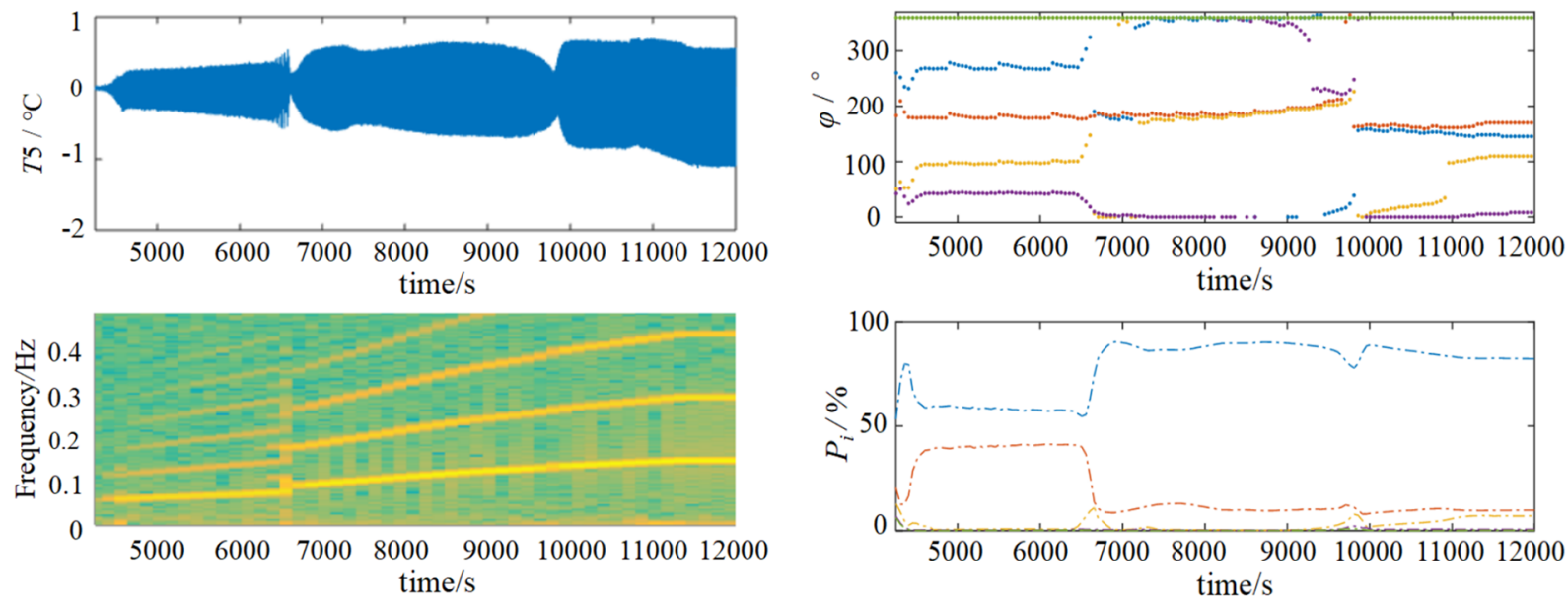

(a)

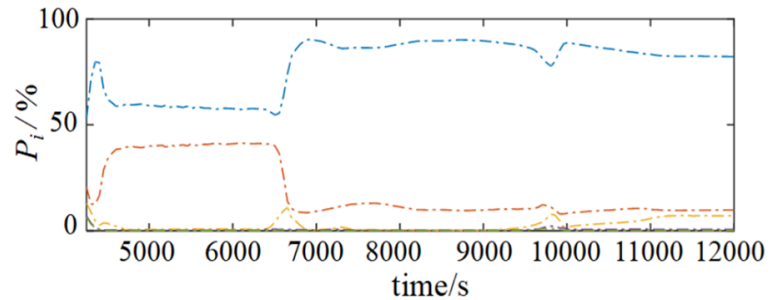

(b)

FIG. 12. Transition process in the liquid bridge at $A r=0.5$ and $V r=0.54$. (a) Temperature signals and time-frequency map. (b) Phase relationship and proportion diagram of principal components.

traveling wave to the standing wave with $m=1$. Figure 12 shows the transition process of thermocapillary convection in a liquid bridge at $A r=0.5$ and $V r=0.54$. The original signal, time-frequency graph, phase diagram, and principal components of $T_{5}$ are shown in the figure. The critical oscillation has characteristics of standing wave with its oscillation phase of about $0^{\circ}$ or $180^{\circ}$, and the proportion of first principal components $\mathrm{P} 1$ is very large, i.e., about $80 \%$. However, the standing wave transforms to a stable traveling wave immediately with the first principal component decreasing to $60 \%$ quickly and phases changing to $269^{\circ}, 179^{\circ}, 95^{\circ}, 44^{\circ}$, and $360^{\circ}$. We consider the critical mode as the stable oscillation after onset, and the transient phenomena of standing wave in the initial stage are not discussed in this paper. Therefore, we believe that the critical oscillation mode is a low-frequency traveling wave with $m=1$.

As shown in Fig. 12, the oscillation wave mode of thermocapillary convection changes obviously when time is at about $6500 \mathrm{~s}$. The oscillation frequency increases abruptly as seen in the timefrequency map, which indicates that the oscillation transits from the low-frequency mode to the high-frequency mode. It is found from the phase diagram that the oscillation phase gradually evolves to $0^{\circ}$ or $180^{\circ}$, and the energy proportion of the first principal component increases to more than $80 \%$, so the wave mode transforms from a traveling wave to a standing wave. The mixed oscillation process when the traveling wave transforms to standing wave can be observed in both phase diagram and proportion diagram of principal components. This mixed oscillation exists when the temperature difference is $25.9^{\circ} \mathrm{C}<\Delta T<27.4^{\circ} \mathrm{C}$. This has also verified the rationality of critical curves in Fig. 8(b). In addition, a second significant change appears in the amplitude when time is at about $9900 \mathrm{~s}$, but it keeps in a standing wave mode with no frequency change. By analysis, this is because of the change in positions of standing wave nodes. Since the positions of standing wave nodes have randomness, the change in positions of wave nodes does not belong to the change of wave mode.

\section{B. Wave mode transformations in liquid bridges at different aspect ratios}

The high-frequency mode appears as a standing wave with $m=1$ no matter it is transformed from the steady state directly in a liquid bridge with a large volume ratio or from the low-frequency mode in a liquid bridge with a small volume ratio. However, for the high-frequency mode, the standing wave is not an absolutely stable oscillation form. When the temperature increases, the highfrequency wave mode has various transformations and evolutions. Experiments show that the transformation process is very sensitive to the aspect ratio; therefore, for liquid bridges at different aspect ratios, we study the volume ratio dependence of transformation process between the traveling wave and the standing wave.

\section{Liquid bridges at $A r=0.5-0.7$}

In order to give a vivid description of oscillation characteristics of oscillatory thermocapillary flow, we show the wave signals of thermocouples T1 and T2 in two periods simultaneously. Since there is a $90^{\circ}$ azimuthal angle between measuring points $\mathrm{T} 1$ and $\mathrm{T} 2$, when the traveling wave with $m=1$ appears, the phase difference between the two signals is $90^{\circ}$, and their amplitudes are close; when the standing wave with $m=1$ appears, the phase difference between them is $0^{\circ}$ or $180^{\circ}$, and their amplitudes exhibit a difference. For example, Fig. 13(a1) shows wave signals at the temperature difference of $\Delta T=16.83^{\circ} \mathrm{C}$, and $T_{1}$ and $T_{2}$ have the same waveform with a phase difference of $90^{\circ}$, so the oscillation is a traveling wave. Figure 13(a2) shows wave signals at the temperature difference of $\Delta T$ $=28.68^{\circ} \mathrm{C}$, and the amplitude of $T_{2}$ is big, but the amplitude of $T_{1}$ is small and the frequency of $T_{1}$ is as twice as that of $T_{2}$. According to analyses in Sec. III B, it is a standing wave oscillation with $m=1$ accompanied by a double-frequency harmonic wave with $m=0$. The amplitude of $T_{1}$ is small because the thermocouple $T 1$ is close to the wave node of standing wave; the amplitude of $T_{2}$ is large because 

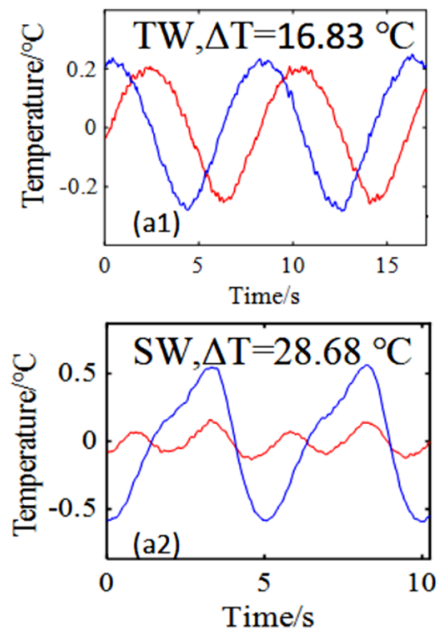

(a)
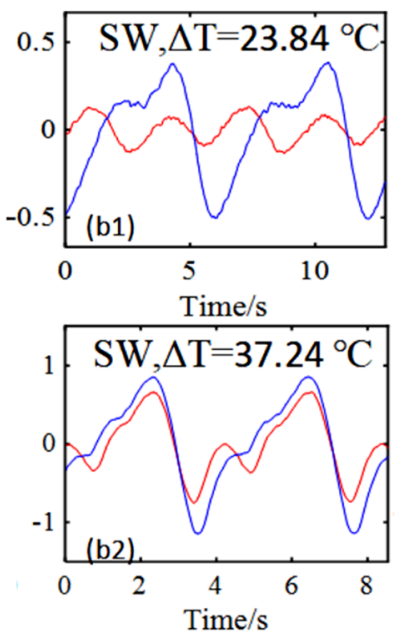

(b)
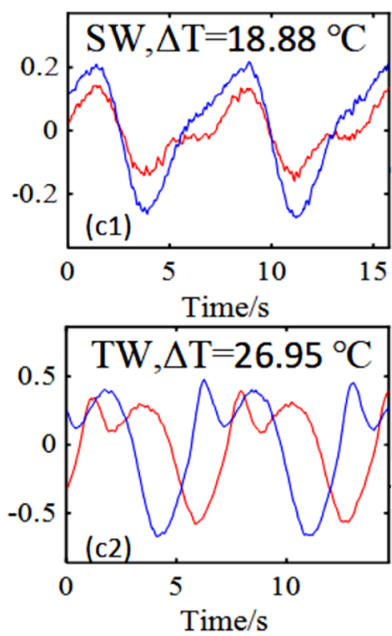

(c)

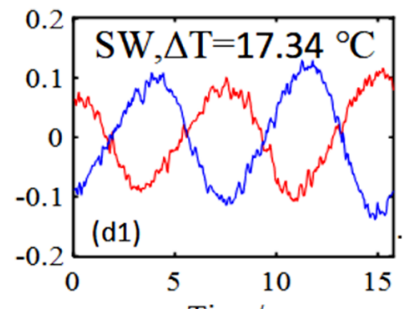

Time/s

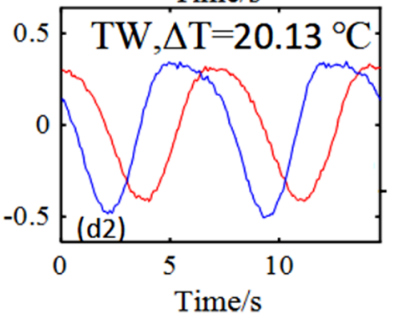

(d)

FIG. 13. Oscillation waveforms at different volume ratios when $A r=0.5$. (a) $V r=0.54$. (b) $V r=0.62$. (c) $V r=0.70$. (d) $V r=0.78$ (red: $T_{1} ;$ blue: $T_{2}$ ).

the thermocouple $\mathrm{T} 2$ is close to the wave loop of standing wave. It is the double-frequency harmonic wave with $m=0$ that makes $T_{1}$ a double-frequency signal. Similarly by analysis, we know that the oscillation in Fig. 13(c) is a traveling wave with $m=1$ accompanied by a double-frequency harmonic wave with $m=2$.

Figure 13 shows waveforms at different temperature differences and volume ratios when the aspect ratio $A r=0.5$. When $V r=0.54$ [Fig. 13(a)], the critical oscillation is a low-frequency traveling wave with $m=1$, and then it transforms to a high-frequency standing wave with $m=1$ accompanied by a double-frequency harmonic wave with $m=0$. When $V r=0.62$ [Fig. 13(b1)] and $V r=0.70$ [Fig. 13(c1)], the critical oscillation is a high-frequency standing wave with $m$ $=1$ accompanied by a double-frequency harmonic wave with $m=0$. When $V r=0.62$, the high-frequency standing wave in the liquid bridge is very stable. When $V r=0.70$, the standing wave mode will transform into the traveling wave mode in the liquid bridge, and it finally develops into a high-frequency traveling wave with $m=1$ accompanied by a double-frequency harmonic wave with $m=2$. As shown in Fig. 13(c2), there are two obvious sub-peaks on wave signals. The wave mode with $m=0$ in the standing wave and the wave mode with $m=2$ in the traveling wave are both double-frequency oscillations. With the increasing of volume ratio, the energy of the double-frequency oscillation decreases gradually. When $V r=0.78$ and 0.90 , the transformation process from standing wave to traveling wave is also observed, but no obvious double-frequency oscillation mode with $m=0$ or $m=2$ is found.

Figure 14 shows the transformation map of supercritical modes when $A r=0.5$. The conditions of the critical waves and the supercritical transitions are represented by three curves. The first curve on the left is a low-frequency traveling wave mode, and it transforms into the standing wave mode with the increase in $M a$ number. The second curve is a high-frequency standing wave mode, and its critical $M a$ number decreases with the increase in volume ratio. With the increase in $M a$ number, the high-frequency mode will transform from a standing wave to a traveling wave, and the
Ma number for transformation shows a decreasing trend with the increase in volume ratio (the third curve). From Fig. 14, one can see that the oscillation region of standing wave is of band type, and the standing wave band narrows down gradually with the increase in volume ratio. Therefore, under the condition of a large volume ratio, the standing wave only appears at the initial stage of oscillation onset, and then it transforms into the traveling wave in a short time.

Liquid bridges at the aspect ratio $A r=0.5-0.7$ exhibit similar transformation processes between the traveling wave and the standing wave. Figure 15 shows the transformation map of supercritical modes when $A r=0.7$. With the increase in aspect ratio, the demarcation volume ratios of the first and second critical curves increase gradually, with curves shifting to the right as a whole. As the aspect ratio increases, the high-frequency oscillation band of traveling wave

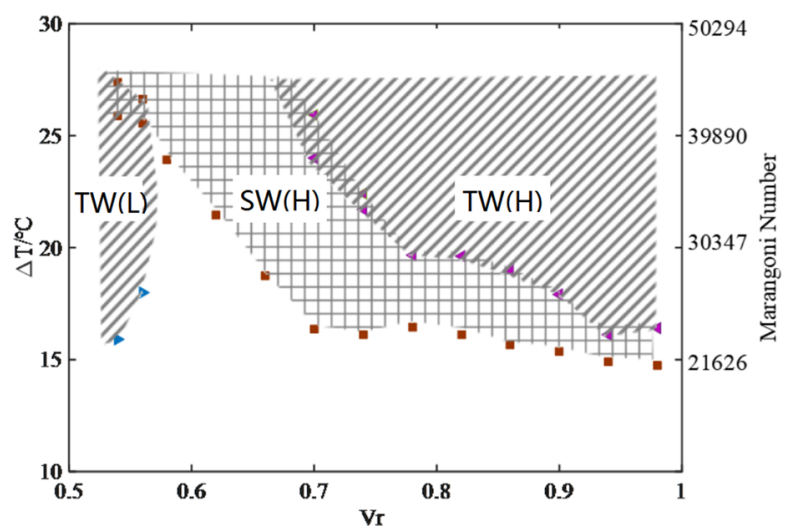

FIG. 14. Transformation map of supercritical modes when $A r=0.5$. TW: traveling wave, SW: standing wave, $(\mathrm{L})$ : low-frequency mode, and $(\mathrm{H})$ : high-frequency mode. 


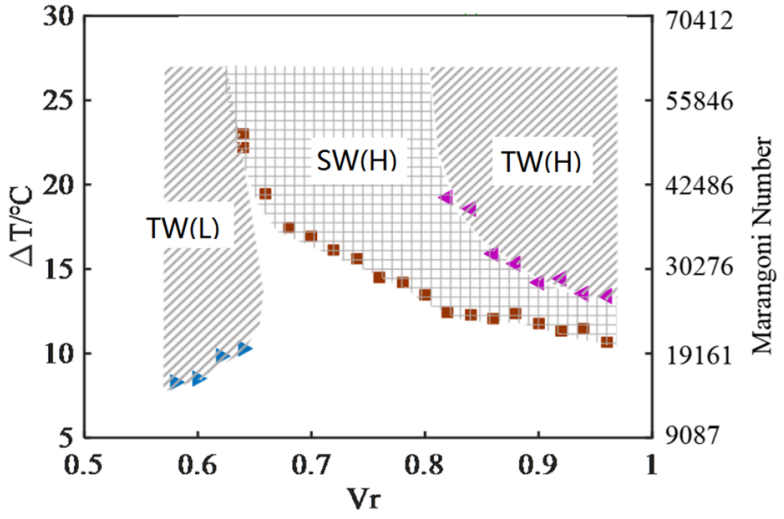

FIG. 15. Transformation map of supercritical modes when $A r=0.7$. TW: traveling wave, SW: standing wave, $(\mathrm{L})$ : low-frequency mode, and $(\mathrm{H})$ : high-frequency mode.

narrows, but the oscillation band of standing wave widens. Therefore, in the range of $A r=0.5-0.7$, the wave transformation process does not change essentially, critical curves shift to the direction of large volume ratio, and the standing wave oscillation mode of the branch of large volume ratios is increasingly stable.

\section{Liquid bridges at $\mathrm{Ar}=0.8$}

Figure 16 shows waveforms at different temperature differences and volume ratios when the aspect ratio $A r=0.8$. In liquid bridges at small volume ratios of $V r=0.56$ and 0.67 [Figs. 16(a) and 16(b)], the critical oscillation of thermocapillary convection is a low-frequency traveling wave with $m=1$ accompanied by a double-frequency harmonic wave with $m=2$. With the increase in temperature difference, the low-frequency traveling wave mode transforms to the high-frequency standing wave mode, and the double-frequency harmonic wave transforms from $m=2$ to $m=0$. The waveform in Fig. 16(a2) shows the quasi-periodicity in the liquid bridge at $V r=0.56$. This is the mixed mode appearing in the transition from low-frequency oscillation mode to high-frequency oscillation mode. Although the oscillation at this time is in a quasiperiodic oscillation state, it is still dominantly a traveling wave mode. In liquid bridges at large volume ratios of $V r=0.81,0.83$ and 0.87 [Figs. 16(c)-16(e)], the critical oscillation of thermocapillary convection is a high-frequency standing wave with $m=1$ accompanied by an obvious double-frequency harmonic wave with $m=0$. For the liquid bridge at $A r=0.8$, the high-frequency standing wave with $m=1$ is a very stable mode, and the phenomenon of a second mode transformation does not appear during the temperature increasing process.

Figure 17 shows the transformation map of supercritical modes when $A r=0.8$. The left branch is a low-frequency traveling wave with $m=1$, and the right branch is a high-frequency standing wave with $m=1$. The low-frequency traveling wave of the left branch will transform into the high-frequency standing wave mode with the increase in $M a$ number, and there exists a mixed oscillation band where the low-frequency mode transits to the high-frequency mode. The mixed oscillation band expands with the decrease in volume ratio; therefore, an obvious mixed oscillation can be observed at $V r=0.61$. Compared with transformation processes in liquid bridges at $\mathrm{Ar}=0.5-0.7$, the demarcation points between the low-frequency mode and the high-frequency mode continues to shift to the right as the aspect ratio increases. The high-frequency standing wave in liquid bridges at $A r=0.5-0.7$ is unstable, and the transformation process to the high-frequency traveling wave appears. However, the oscillation region increasing of the high-frequency traveling wave shows a shrinking trend with $A r$. In liquid bridges at $A r=0.8$, the disappearance of oscillation region of the traveling wave conforms to this trend exactly. Therefore, when $A r=0.5-0.8$, the high-frequency standing wave with $m=1$ is increasingly stable as the aspect ratio increases.

A special phenomenon appears in the liquid bridge at $A r=0.8$, which is the jump phenomenon on the critical curve at $V r=0.82$. The critical modes of the two oscillations are compared in Figs. 16(c1) and 16(d1), and it is found that both of them are
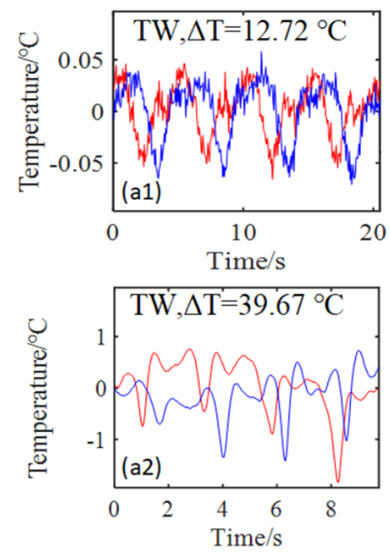

(a)
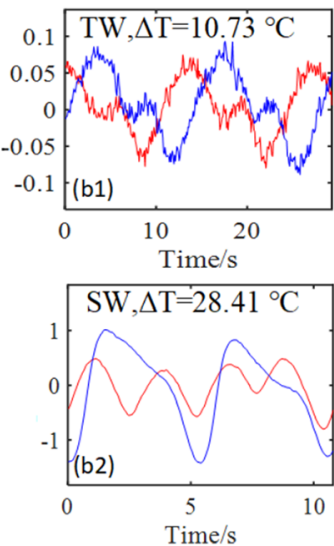

(b)
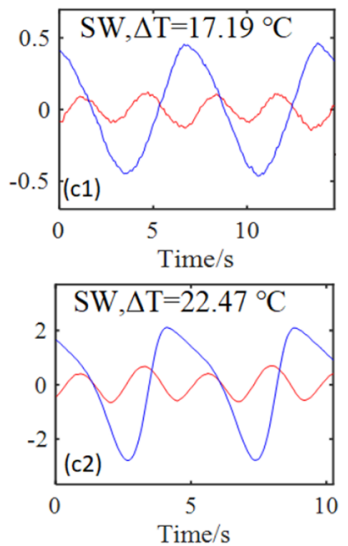

(c)
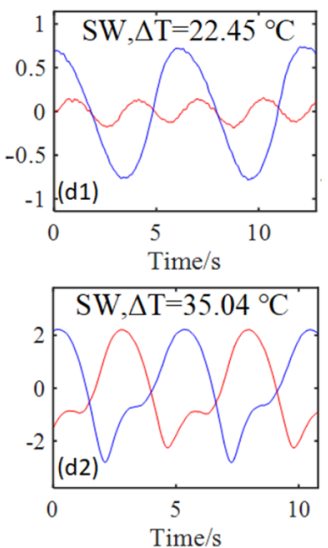

(d)
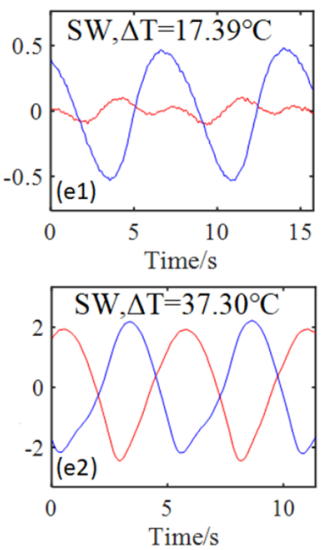

(e)

FIG. 16. Oscillation waveforms at different volume ratios when $A r=0.8$. (a) $V r=0.56$. (b) $V r=0.67$. (c) $V r=0.81$. (d) $V r=0.83$. (e) $V r=0.97$ (red: $T_{1}$; blue: $T_{2}$ ). 


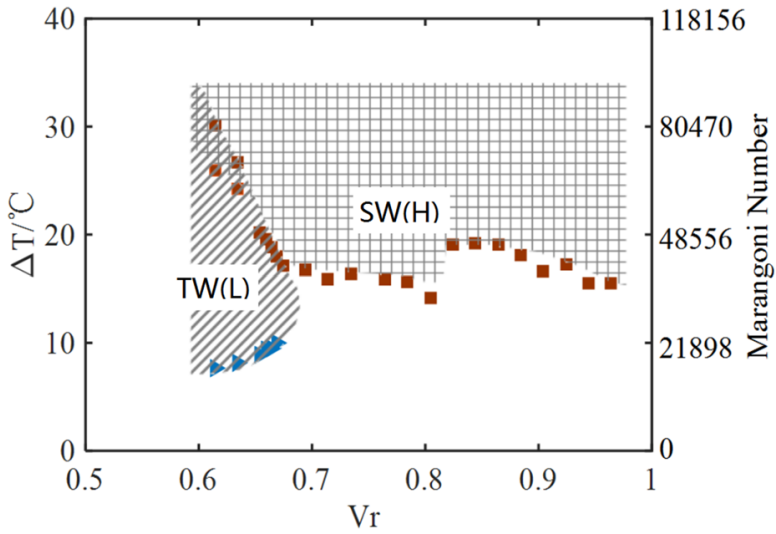

FIG. 17. Transformation map of supercritical modes when $A r=0.8$. TW: traveling wave, SW: standing wave, $(\mathrm{L})$ : low-frequency mode, and $(\mathrm{H})$ : high-frequency mode.

the typical standing wave mode with $m=1$, with positions of wave nodes at $\mathrm{T} 1$ and $\mathrm{T} 3$. The critical temperature difference at $V r=0.81$ is $15.4^{\circ} \mathrm{C}$, while the critical temperature difference at $V r=0.83$ is $19.0^{\circ} \mathrm{C}$. Although the critical condition changes abruptly, these two oscillations correspond to the same mode. Near the breakpoint at $V r=0.83$, we also observed the phenomenon that the oscillation of thermocapillary convection disappears after the onset and then starts again.

\section{Liquid bridges at $\mathrm{Ar}=0.9$}

Figure 18 shows waveforms at different temperature differences and volume ratios when the aspect ratio $A r=0.9$. The critical oscillations at $V r=0.61$ and $V r=0.69$ are the traveling wave with $m=1$ accompanied by a double-frequency mode with $m=2$. The flow field in the liquid bridge at $V r=0.61$ enters a disordered state after the temperature difference is increased, and it is not easy to judge its characteristics as traveling wave or standing wave, as shown in Fig. 20(a2). Increasing the temperature difference until the flow field enters an orderly oscillation state. As shown in Fig. 20(a3), the waveform is in a standing wave state. At $V r$ $=0.69$, where the volume ratio is close to the demarcation point, the low-frequency traveling wave with $m=1$ transforms to the highfrequency standing wave with $m=1$ directly, being accompanied by the double-frequency oscillation with $m=0$. The critical oscillations at $V r=0.71$ and $V r=0.81$ are the stable standing wave mode with $m=1$, and the double-frequency oscillation at $V r=0.81$ [Fig. 20(c1)] is much lower than the double-frequency oscillation at $V r=0.69$ [Fig. 20(b1)]. The critical oscillation at $V r=0.89$ shows characteristics of standing wave temporarily, and it develops into a traveling wave mode when stable. As shown in Fig. 20(e2), $T_{1}$ and $T_{2}$ are not exactly synchronous, and the phase difference between them is $59^{\circ}$. This is not a standard standing wave mode or traveling wave mode, so two thermocouple signals are not enough for the judgment and it is necessary to analyze phases of all five thermocouple signals together. The phases of $T_{1}-T_{5}$ are $115^{\circ}, 174^{\circ}, 316^{\circ}, 342^{\circ}$, and $360^{\circ}$, and the phase of $T_{1}-T_{2}$ and the phase of $T_{3}-T_{5}$ have a difference about $180^{\circ}$. We judge that the oscillation is dominantly a
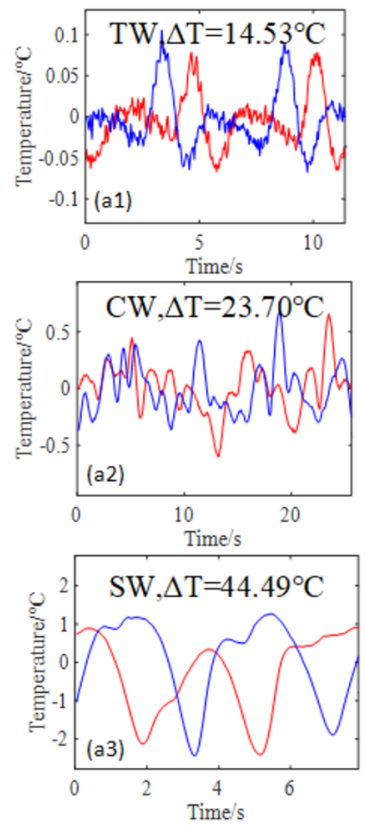

(a)
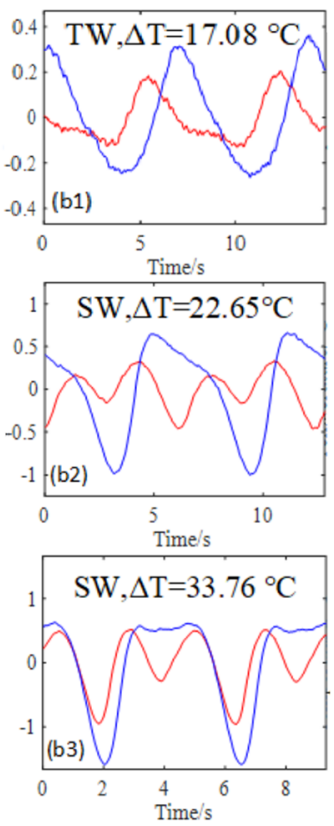

(b)
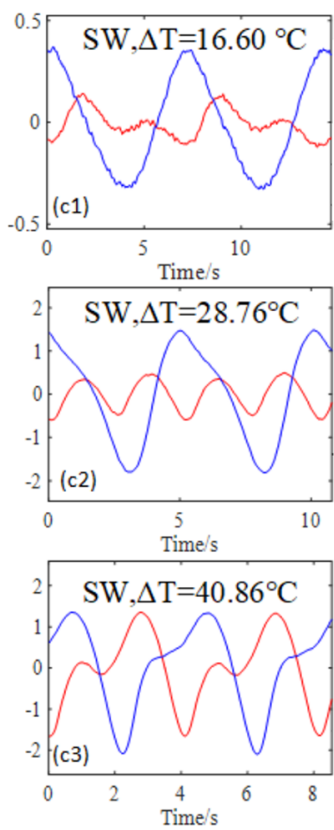

(c)
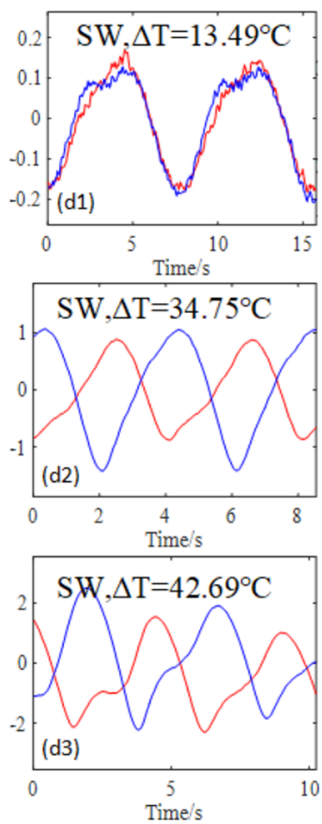

(d)
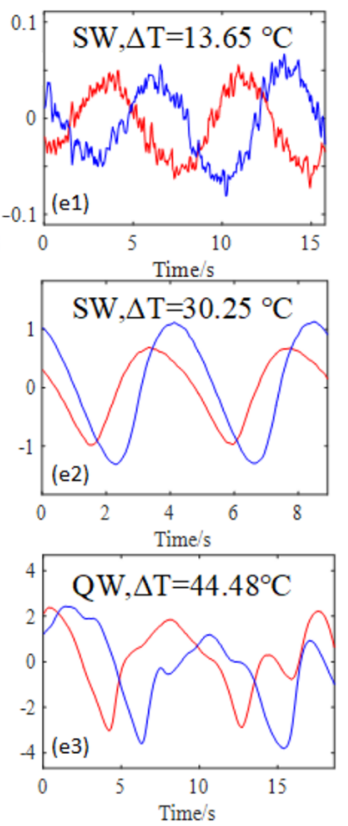

(e)

FIG. 18. Oscillation waveforms at different volume ratios when $A r=0.9$. (a) $V r=0.61$. (b) $V r=0.69$. (c) $V r=0.71$. (d) $V r=0.81$. (e) $V r=0.89$ (red: $T_{1}$, blue: $T_{2}$ ). 


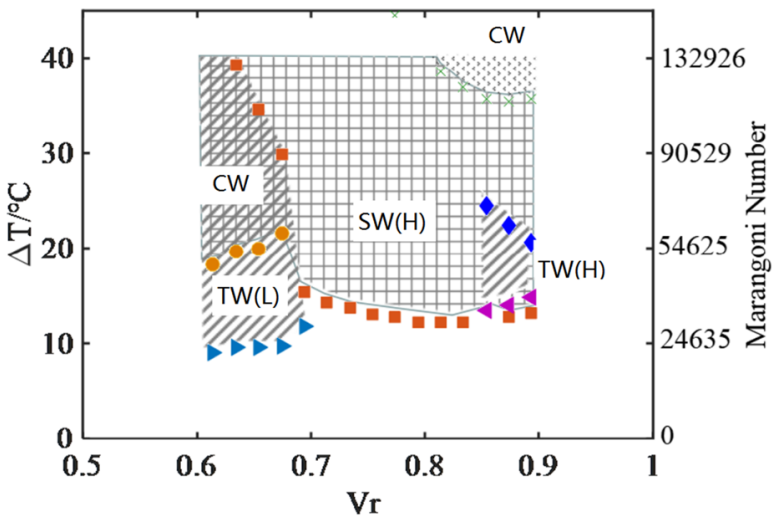

FIG. 19. Transformation map of supercritical modes when $A r=0.9$. TW: traveling wave, SW: standing wave, CW: chaotic wave, $(\mathrm{L})$ : low-frequency mode, and $(\mathrm{H})$ : high-frequency mode.

standing wave mode with certain characteristics of traveling wave. [Fig. 20(e2)].

Figure 19 shows the transformation map of supercritical modes when $A r=0.9$. In the liquid bridges at $A r=0.9$, the chaotic wave appears in the regions of small volume ratio and the traveling wave dominated oscillation appear in the regions of large volume ratio. When $V r<0.69$, as the $M a$ number is further increased, the critical traveling wave can easily transform into the irregular oscillation at low $M a$ number $\left(M a>2 \Delta M a_{c}\right)$ and then to the standing wave. This transition process is: traveling wave (lowfrequency) $\rightarrow$ chaotic wave $\rightarrow$ standing wave (high-frequency). The high-frequency oscillation of the fat liquid bridge branch is a stable standing wave mode when $V r=0.70-0.83$; but when $V r>$ 0.83 , the high-frequency standing wave transforms to the traveling wave immediately after its appearance, passing a very narrow highfrequency traveling wave region, then transforms to the standing wave.

\section{Liquid bridges at $\mathrm{Ar}=1.0$}

Figure 20 shows waveform at different temperature differences and volume ratios when the aspect ratio $A r=1.0$. In Figs. 20(a)20(b), the critical mode in liquid bridges at $V r=0.56$ and $V r$ $=0.68$ is the low-frequency traveling wave with $m=1$ accompanied by a double-frequency harmonic wave with $m=2$. With the increase in temperature difference, distinct supercritical transformation processes appear in the two liquid bridges. When $V r=0.56$, the wave mode transformation process is traveling wave (low-frequency) $\rightarrow$ standing wave (high-frequency) $\rightarrow$ chaotic wave; when $V r=0.68$, the wave mode transformation process is low-frequency traveling wave $\rightarrow$ chaotic wave $\rightarrow$ high-frequency standing wave. When $V r$ $=0.72,0.88$, and 0.96 , as shown in Figs. 20 (c) $-20(\mathrm{e})$, the critical oscillation mode is the high-frequency standing wave with $m=1$. With the increase in temperature difference, the high-frequency standing wave modes in the three liquid bridges have the same transformation process: standing wave (high-frequency) $\rightarrow$ traveling wave (high-frequency) $\rightarrow$ standing wave (high-frequency) [in Figs. 20 (c)$20(\mathrm{e})]$. When $V r=0.72$ or 0.88 , both the critical standing wave mode and the standing wave mode after three transitions are coupled with the double-frequency signal with $m=0$, and the energy of a double-frequency harmonic wave decreases with the increase in volume ratio. When $V r=0.96$, the oscillation waveform is close to
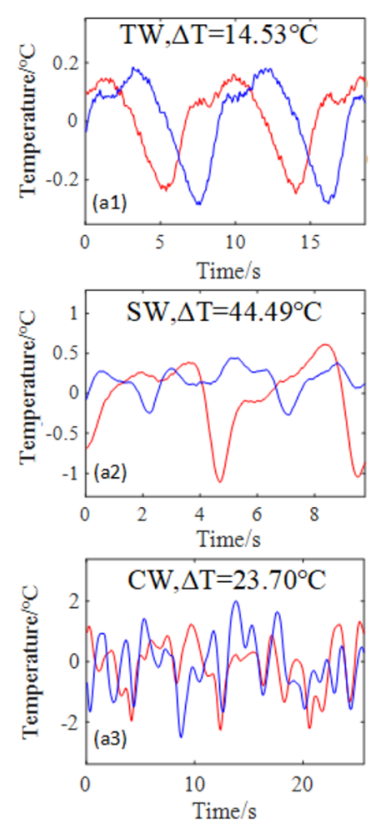

(a)
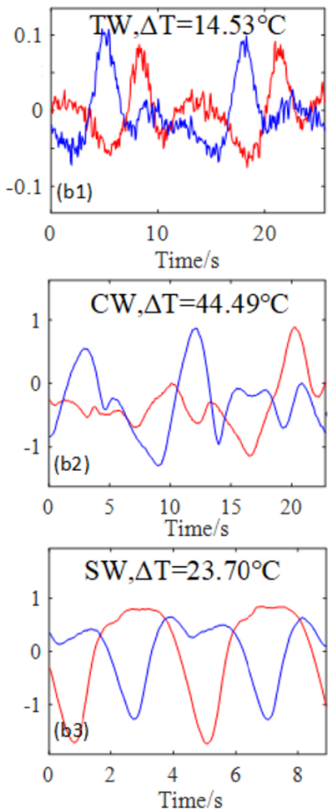

(b)
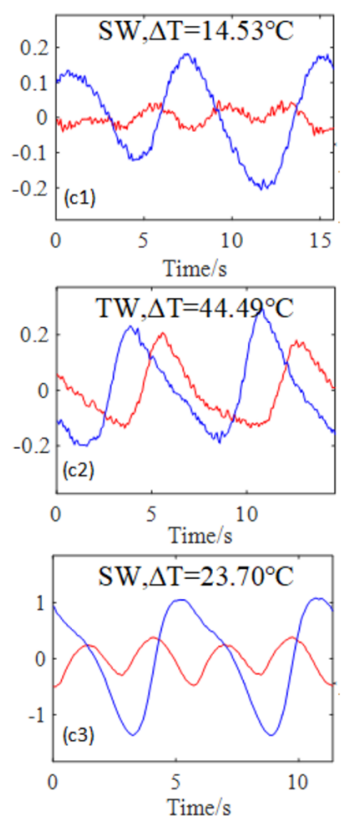

(c)
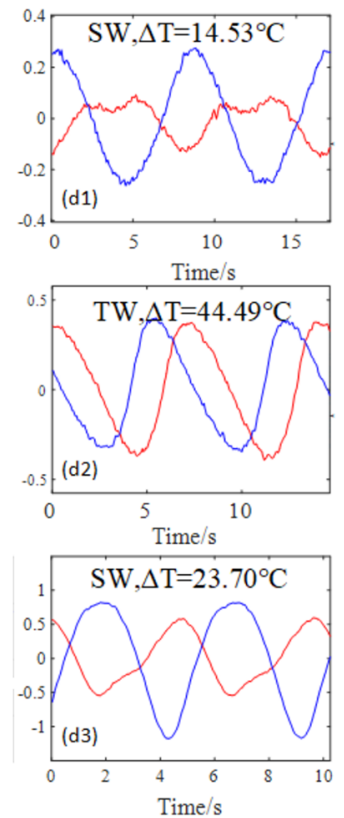

(d)
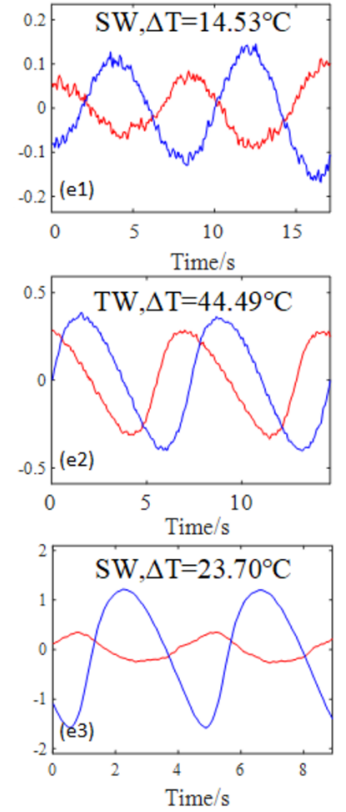

(e)

FIG. 20. Oscillation waveforms at different volume ratios when $A r=1.0$. (a) $V r=0.56$. (b) $V r=0.68$. (c) $V r=0.72$. (d) $V r=0.88$. (e) $V r=0.96$ (red: $T_{1}$; blue: $T_{2}$ ). 


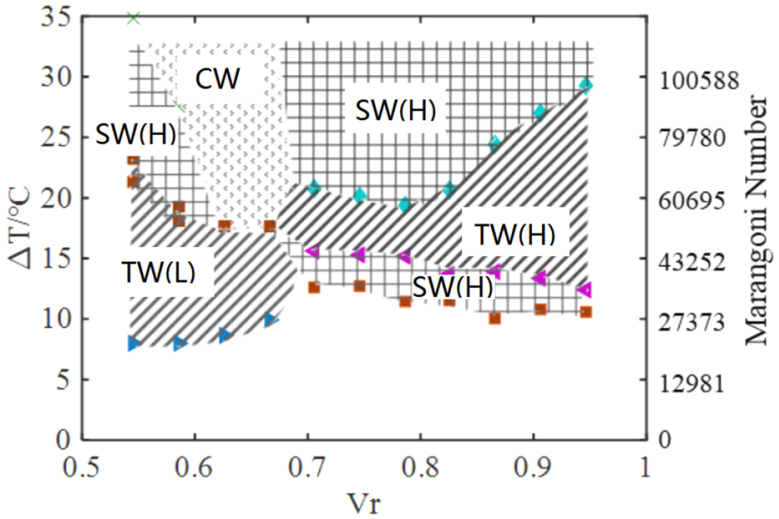

FIG. 21. Transformation map of supercritical modes when $A r=1.0$. TW: traveling wave, SW: standing wave, CW: chaotic wave, $(\mathrm{L})$ : low-frequency mode, and $(\mathrm{H})$ : high-frequency mode.

the sinusoidal signal, and no obvious double-frequency harmonic is observed.

Figure 21 shows the transformation map of supercritical modes when $A r=1.0$. In the liquid bridge at a small volume ratio, there exist the traveling wave band, standing wave band, and chaotic wave band. When $V r \leq 0.58$, thermocapillary oscillation transforms as traveling wave $\rightarrow$ standing wave $\rightarrow$ irregular wave. When $V r$ $\geq 0.62$, thermocapillary oscillation transforms as traveling wave $\rightarrow$ irregular wave. The irregular wave transforms to the standing wave near the demarcation point. In the liquid bridge at a large volume ratio, the standing wave band, traveling wave band, and standing wave band appear in turn as the $M a$ number increases. The critical oscillation is a narrow standing wave oscillation band, so the high-frequency mode appears as a standing wave mode first and then transforms to the traveling wave mode. With a further increase in $M a$ number, the traveling wave mode will transform into the standing wave mode. The critical condition for the transformation from a high-frequency traveling wave to a high-frequency standing wave shows a trend of decreasing first and then increases with the increase in volume ratio. When $V r>0.8$, the traveling wave band widens significantly with the increase in volume ratio, and the traveling wave is more stable under the condition of large volume ratio.

\section{CONCLUSIONS}

As there are two zones of different critical frequencies on the $V r-A r$ plane in our space experiments, ${ }^{26}$ we have investigated the wave characteristics and the supercritical transitions in this paper. The low-frequency mode happens in the zone with a small volume ratio or a large aspect ratio; the high-frequency mode happens in the zone with a large volume ratio or a small aspect ratio. Both azimuthal wavenumbers in these zones are $m=1$, but the wave characteristics are different. In the critical condition, the low-frequency mode is a traveling wave $(m=1)$ and the high-frequency mode is a standing wave $(m=1)$.

Two types of supercritical transitions are found with the increasing of $M a$ : (1) transitions of frequency, that is, the low-frequency mode transits to the high-frequency mode; (2) wave transformations without frequency transition, that is, the wave changes its mode among traveling waves, standing waves, and chaotic waves. The change of azimuthal wave number is not found during the supercritical process. The transition maps of $A r=0.5-$ 1.0 have been given in Figs. 14, 15, 17, 19, and 21. Details of these transition processes will be discussed as follows.

Transitions of frequency happen at the left branch (small volume ratio) of the critical curve, as shown in Fig. 8(b). Since the right branch (large volume ratio) of the critical curve extends to the small volume ratio, it results in transitions from a low-frequency mode to a high-frequency mode. The frequency transition corresponds to the transformation from a traveling wave ( $m=1$, low-frequency) to a standing wave ( $m=1$, high-frequency). In liquid bridges at $A r=0.8$ and $A r=1.0$, the chaotic wave is observed, and the transition process is as follows: traveling wave $(m=1$, low-frequency) $\rightarrow$ chaotic wave $\rightarrow$ sanding wave ( $m=1$, high-frequency). Since the transition process, traveling wave ( $m=1$, low-frequency) $\rightarrow$ standing wave ( $m=1$, high-frequency) $\rightarrow$ chaotic wave, has also been observed, we believe that this kind of chaotic wave is not the mixed mode during the transition process from a traveling wave to a standing wave.

Wave transformations without frequency transition occur when the Marangoni number $M a$ is beyond the right branch (large volume ratio) of the critical curve. Without the transition of frequency, the characteristic of wave changes with the $M a$ increasing. The most commonly observed wave transformation is the critical standing wave ( $m=1$, high-frequency) $\rightarrow$ supercritical traveling wave ( $m=1$, high-frequency). It can be obtained from the transition map that the stable region of a standing wave is a band. When the aspect ratio increases from $A r=0.5-0.7$, the standing wave band expands and the transformation into a traveling wave is absent in some small volume ratio. When the aspect ratio increases to $A r=0.8$, the high-frequency standing wave mode is very stable and no traveling wave mode appears. When $A r=0.9$, the traveling wave band appears from the right side $(V r>0.83)$. When $A r=1.0$, this oscillation band extends to the entire right branch, and the wave mode transformations are standing wave ( $m=1$, high-frequency) $\rightarrow$ traveling wave $(m=1$, high-frequency) $\rightarrow$ standing wave $(m=1$, high-frequency).

Two types of double-frequency waves, which $m=0$ and $m=2$, appear as harmonic waves in the small volume ratio case. The wave mode with $m=0$ and the traveling wave mode with $m=2$ are doublefrequency harmonic components that superimposed the standing wave with $m=1$ and the traveling wave with $m=1$, respectively. The energy of this harmonic mode increases with the decrease of volume ratio. These new harmonic modes have never been reported before, probably because that they are hardly observed in the liquid bridge of a large volume ratio, $V r \approx 1$; however, most experiments have been carried out on liquid bridges at $V r \approx 1$.

For comparison, we have finished a ground experiment with a similar set-up; 2 cSt silicone oil $(P r=28)$ has been used as the fluid media; the wave regimes have been investigated in a short liquid bridge. ${ }^{18}$ The wave regimes are standing waves $\left(1 \leq M a / M a_{c}<1.2\right)$, traveling wave $\left(1.2 \leq M a / M a_{\mathrm{c}}<1.9\right)$, local two wave separation (1.0 $\leq$ $\left.M a / M a_{c}<2.1\right)$, and chaotic waves $\left(M a / M a_{c} \geq 2.1\right)$. To some extent, these transformation processes show similarity with space experiment results $(\operatorname{Pr}=67)$. However, the supercritical transformation is 
very sensitive to the geometry of a liquid bridge. Our space experiment gives clear maps of supercritical transformation dependent on aspect ratios and volume ratios for the first time. According to the details of the transformation maps, new phenomena or conclusions are obtained:

(1) In the high-frequency zone, the transformation from the critical standing wave to the traveling wave occurs.

(2) In the low-frequency zone, the transition of frequency leads to the transformation from the critical traveling wave to the standing wave.

(3) When $A r=0.7-0.9$, there is a certain band of volume ratio in which the critical standing waves are very stable.

(4) Supercritical transformations of "standing wave $\rightarrow$ traveling wave $\rightarrow$ standing wave" are found when $A r=1.0$.

(5) In most cases, the transition condition $\left(M a / M a_{\mathrm{c}}\right)$ to chaos is estimated to be higher than 3; however, when $A r=0.9-1.0$, the low-frequency mode (the branch of small volume ratio) transits to chaos at a low transition condition, $M a / M a_{\mathrm{c}} \approx 2$.

\section{AUTHOR'S CONTRIBUTIONS}

Q.K. and D.W. contributed equally to this work.

\section{ACKNOWLEDGMENTS}

This project was financially supported by the space experiment project on Tiangong-2 (TG-2) of manned space program of China.

The authors gratefully acknowledge the Technology and Engineering Center for Space Utilization (CSU), CAS, for managing and supporting the space experiment, Xiang Li from CSU, CAS, for designing the electronic control unit of the payload, Lujun $\mathrm{Li}$, who graduated from their research group, for the contribution in the early stage of the work, Longsheng Duan for participating in the monitoring of the space experiments, and Pu Zhang, Shuoting Zhang, Shangtong Chen, and Wenhui Cao for editing the command of experiment programs.

\section{REFERENCES}

${ }^{1}$ D. Schwabe, A. Scharmann, F. Preisser et al., "Experiments on surface tension driven flow in floating zone melting," J. Cryst. Growth 43(3), 305-312 (1978).

${ }^{2} \mathrm{C}$. H. Chun and W. Wuest, "Experiments on the transition from the steady to the oscillatory Marangoni-convection of a floating zone under reduced gravity effect," Acta Astronaut. 6(9), 1073-1082 (1979).

${ }^{3}$ M. K. Smith and S. H. Davis, "Instabilities of dynamic thermocapillary liquid layers. Part 1. Convective instabilities," J. Fluid Mech. 132, 145-162 (1983).

${ }^{4}$ J.-J. Xu and S. H. Davis, "Convective thermocapillary instabilities in liquid bridges," Phys. Fluids 27(5), 1102-1107 (1984).

${ }^{5}$ D. Schwabe, "Hydrothermal waves in a liquid bridge with aspect ratio near the Rayleigh limit under microgravity," Phys. Fluids 17(11), 112104 (2005).

${ }^{6}$ B. Xun, P. G. Chen, K. Li et al., "A linear stability analysis of large-Prandtlnumber thermocapillary liquid bridges," Adv. Space Res. 41(12), 2094-2100 (2008).

${ }^{7}$ R. Velten, D. Schwabe, and A. Scharmann, "The periodic instability of thermocapillary convection in cylindrical liquid bridges," Phys. Fluids A 3(2), 267 (1991).
${ }^{8}$ D. E. Melnikov, V. M. Shevtsova, and J. C. Legros, "Onset of temporal aperiodicity in high Prandtl number liquid bridge under terrestrial conditions," Phys. Fluids 16(5), 1746 (2004).

${ }^{9}$ V. Shevtsova, D. E. Melnikov, and A. Nepomnyashchy, "New flow regimes generated by mode coupling in buoyant-thermocapillary convection," Phys. Rev. Lett. 102(13), 134503 (2009).

${ }^{10} \mathrm{~S}$. Frank and D. Schwabe, "Temporal and spatial elements of thermocapillary convection in floating zones," Exp. Fluids 23(3), 234-251 (1997).

${ }^{11}$ I. I. Ryzhkov, "Thermocapillary instabilities in liquid bridges revisited," Phys. Fluids 23(8), 082103 (2011).

${ }^{12}$ F. Preisser, D. Schwabe, and A. Scharmann, "Steady and oscillatory thermocapillary convection in liquid columns with free cylindrical surface," J. Fluid Mech. 126(126), 545-567 (1983).

${ }^{13}$ T. Yano, K. Nishino, H. Kawamura et al., "Instability and associated roll structure of Marangoni convection in high Prandtl number liquid bridge with large aspect ratio," Phys. Fluids 27(2), 024108 (2015).

${ }^{14} \mathrm{~T}$. Yano, K. Nishino, I. Ueno et al., "Sensitivity of hydrothermal wave instability of Marangoni convection to the interfacial heat transfer in long liquid bridges of high Prandtl number fluids," Phys. Fluids 29(4), 044105 (2017).

${ }^{15} \mathrm{~W}$. R. Hu, J. Z. Shu, R. Zhou et al., "Influence of liquid bridge volume on the onset of oscillation in floating zone convection I. Experiments," J. Cryst. Growth 142(3-4), 379-384 (1994).

${ }^{16}$ Q. S. Chen and W. R. Hu, "Influence of liquid bridge volume on instability of floating half zone convection," Int. J. Heat Mass Transfer 41(6), 825-837 (1998).

${ }^{17}$ B. Xun, K. Li, and W. R. Hu, "Effect of volume ratio on thermocapillary flow in liquid bridges of high-Prandtl-number fluids," Phys. Rev. E: Stat., Nonlinear, Soft Matter Phys. 81(2), 036324 (2010).

${ }^{18} \mathrm{~J}$. Wang, D. Wu, L. Duan, and Q. Kang, "Ground experiment on the instability of buoyant-thermocapillary convection in large-scale liquid bridge with large Prandtl number," Int. J. Heat Mass Transfer 108, 2107-2119 (2017).

${ }^{19}$ Q. Kang, H. Jiang, L. Duan, C. Zhang, and W. Hu, "The critical condition and oscillation - transition characteristics of thermocapillary convection in the space experiment on SJ-10 satellite," Int. J. Heat Mass Transfer 135, 479-490 (2019).

${ }^{20}$ Q. Kang, J. Wang, L. Duan, Y. Y. Su, J. W. He, D. Wu, and W. R. Hu, "The volume ratio effect on flow patterns and transition processes of thermocapillary convection,” J. Fluid Mech. 868, 560-583 (2019).

${ }^{21}$ K. Qi, D. Wu, L. Duan, J. He, L. Hu, L. Duan, and W. Hu, "Surface configurations and wave patterns of thermocapillary convection onboard the SJ10 satellite," Phys. Fluids 31, 044105 (2019).

${ }^{22}$ M. K. Ermakov, "Onset of oscillations in high-prandtl thermocapillary liquid bridges: Linear-stability analysis vs. experiment," in XXI International Congress of Theoretical and Applied Mechanics, Warsaw, Poland, August 15-21, 2004.

${ }^{23}$ V. M. Shevtsova, M. Mojahed, D. E. Melnikov et al., "The choice of the critical mode of hydrothermal instability in liquid bridge," in Interfacial Fluid Dynamics and Transport Processes (Springer, Berlin, Heidelberg, 2003).

${ }^{24}$ B. C. Sim and A. Zebib, "Thermocapillary convection in liquid bridges with undeformable curved surfaces," J. Thermophys. Heat Transfer 16(4), 553-561 (2012).

${ }^{25}$ J. Masud, Y. Kamotani, and S. Ostrach, "Oscillatory thermocapillary flow in cylindrical columns of high Prandtl number fluids," J. Thermophys. Heat Transfer 11(1), 105-111 (2012).

${ }^{26}$ Q. Kang, D. Wu, L. Duan, L. Hu, J. Wang, P. Zhang, and W. Hu, "The effects of geometry and heating rate on thermocapillary convection in the liquid bridge," J. Fluid Mech. 881, 951-982 (2019).

${ }^{27}$ V. Shevtsova, A. Mialdun, H. Kawamura et al., "Onset of hydrothermal instability in liquid bridge. Experimental benchmark," Fluid Dyn. Mater. Process. 7(1), $1-28$ (2011).

${ }^{28}$ L. B. S. Sumner, G. P. Neitzel, and J. P. Fontaine, "Oscillatory thermocapillary convection in liquid bridges with highly deformed free surfaces: Experiments and energy-stability analysis," Phys. Fluids 13(1), 107-120 (2001).

${ }^{29}$ M. Sakurai, N. Ohishi, and A. Hirata, "Effect of liquid bridge form on oscillatory thermocapillary convection under $1 \mathrm{~g}$ and $\mu \mathrm{g}$ conditions," Acta Astronaut. 55(12), 977-983 (2004). 